\title{
POLES OF ARCHIMEDEAN ZETA FUNCTIONS FOR ANALYTIC MAPPINGS
}

\author{
E. LEÓN-CARDENAL, WILLEM VEYS, AND W. A. ZÚÑIGA-GALINDO
}

\begin{abstract}
Let $\boldsymbol{f}=\left(f_{1}, \ldots, f_{l}\right): U \rightarrow K^{l}$, with $K=\mathbb{R}$ or $\mathbb{C}$, be a $K$-analytic mapping defined on an open set $U \subset K^{n}$, and let $\Phi$ be a smooth function on $U$ with compact support. In this paper, we give a description of the possible poles of the local zeta function attached to $(\boldsymbol{f}, \Phi)$ in terms of a log-principalization of the ideal $\mathcal{I}_{\boldsymbol{f}}=\left(f_{1}, \ldots, f_{l}\right)$. When $\boldsymbol{f}$ is a non-degenerate mapping, we give an explicit list for the possible poles of $Z_{\Phi}(s, \boldsymbol{f})$ in terms of the normal vectors to the supporting hyperplanes of a Newton polyhedron attached to $\boldsymbol{f}$, and some additional vectors (or rays) that appear in the construction of a simplicial conical subdivision of the first orthant. These results extend the corresponding results of Varchenko to the case $l \geq 1$, and $K=\mathbb{R}$ or $\mathbb{C}$. In the case $l=1$ and $K=\mathbb{R}$, Denef and Sargos proved that the candidates poles induced by the extra rays required in the construction of a simplicial conical subdivision can be discarded from the list of candidate poles. We extend the Denef-Sargos result arbitrary $l \geq 1$. This yields in general a much shorter list of candidate poles, that can moreover be read off immediately from $\Gamma(\boldsymbol{f})$.
\end{abstract}

\section{INTRODUCTION}

We take $K=\mathbb{R}$ or $\mathbb{C}$. Let $\boldsymbol{f}=\left(f_{1}, \ldots, f_{l}\right): U \rightarrow K^{l}$ be a $K$-analytic mapping defined on an open $U$ in $K^{n}$. Let $\Phi: U \rightarrow \mathbb{C}$ be a smooth function on $U$ with compact support. Then the local zeta function attached to $(\boldsymbol{f}, \Phi)$ is defined as

$$
Z_{\Phi}(s, \boldsymbol{f})=\int_{K^{n} \backslash \boldsymbol{f}^{-1}(0)} \Phi(x)|\boldsymbol{f}(x)|_{K}^{s}|d x|,
$$

for $s \in \mathbb{C}$ with $\operatorname{Re}(s)>0$, where $|d x|$ is the Haar measure on $K^{n}$. The local zeta functions have a meromorphic continuation to the whole complex plane. In the case $l=1$, the meromorphic continuation of $Z_{\Phi}(s, \boldsymbol{f})$ was established jointly by Bernstein and Gel'fand [3], independently by Atiyah [2], then by a different method by Bernstein [4]. In [10], see also [11], Igusa developed a uniform theory for local zeta functions over local fields of characteristic zero. In this context, there exist asymptotic expansions for oscillatory integrals depending on one parameter which are controlled by the poles of 'twisted versions' of $Z_{\Phi}(s, \boldsymbol{f})$, see also [1], [20]. In [18], with $l \geq 1$ and $K=\mathbb{C}$, Phong and Sturm studied the stability of the poles of $Z_{\Phi}(s, \boldsymbol{f})$ under small perturbations of $\boldsymbol{f}$.

In this paper, we give a geometric description of the possible poles of $Z_{\Phi}(s, \boldsymbol{f})$, including the largest one, in terms of a log-principalization of the ideal $\mathcal{I}_{\boldsymbol{f}}=$

1991 Mathematics Subject Classification. Primary: 11M41, Secondary: 32S05, 14B05, 14 M25.

Key words and phrases. Local zeta functions, analytic mappings, log canonical threshold, logprincipalization of ideals, Newton polyhedra, toric varieties, decoupages.

The third author was partially supported by Conacyt, Grant \# 127794. 
$\left(f_{1}, \ldots, f_{l}\right)$, see Theorem 2 and Proposition 2. To the best of our knowledge a such description has not been reported before, in the Archimedean context. (See however Remark 1 and Proposition 1 when $K=\mathbb{R}$.) When $\boldsymbol{f}$ is a non-degenerate mapping in the sense of [21], we give an explicit list for the possible poles of $Z_{\Phi}(s, \boldsymbol{f})$ in terms of the normal vectors to the supporting hyperplanes of a Newton polyhedron attached to $\boldsymbol{f}$, and additional vectors (or rays) that appear in the construction of a simplicial conical subdivision of the first orthant, see Theorem 3. These results extend the corresponding results of Varchenko in [20] for $l=1$ and $K=\mathbb{R}$ to the case $l \geq 1$, and $K=\mathbb{R}$ or $\mathbb{C}$. In the case $l=1$ and $K=\mathbb{R}$, Denef and Sargos proved in [5] a strong and interesting result: the candidate poles induced by the extra rays required in the construction of a simplicial conical subdivision can be discarded from the list of candidate poles in Theorem 3. We extend the Denef-Sargos result to arbitrary $l \geq 1$, see Theorems 4,5 , and 6 . This yields in general a much shorter list of candidate poles, that can moreover be read off immediately from $\Gamma(\boldsymbol{f})$.

At this point we must mention that in the case $K=\mathbb{R}$ a description of the poles of $Z_{\Phi}(s, \boldsymbol{f})$ can be obtained by using an embedded resolution of singularities of $\sum_{i=1}^{l} f_{i}^{2}$, see [1], [11], [20]. In particular, one could define $\boldsymbol{f}$ to be non-degenerate as meaning $\sum_{i=1}^{l} f_{i}^{2}$ to be non-degenerate in the usual sense, and thus one could use all the results of [20]. We note however that there exist many mappings $\boldsymbol{f}$ which are non-degenerate in the sense of our Definition 1 but such that $\sum_{i=1}^{l} f_{i}^{2}$ is degenerate in the usual sense, see Remark 4. Thus, our approach gives a finer explicit description of the poles of $Z_{\Phi}(s, \boldsymbol{f})$.

\section{Local Zeta Functions for Analytic Mappings}

2.1. Fixing the data. We take $K=\mathbb{R}$ or $\mathbb{C}$. For $a=\left(a_{1}, \ldots, a_{n}\right) \in K^{n}$ we put $|a|_{K}=|a|_{\mathbb{R}}$ or $|a|_{\mathbb{C}}^{2}$, where $|\cdot|_{\mathbb{R}}$ and $|\cdot|_{\mathbb{C}}$ are the standard norms on $\mathbb{R}^{n}$ and $\mathbb{C}^{n}$, respectively.

Let $f_{1}, \ldots, f_{l}$ be polynomials in $K\left[x_{1}, \ldots, x_{n}\right]$, or, more generally, $K$-analytic functions on an open set $U \subset K^{n}$. We consider the mapping $\boldsymbol{f}=\left(f_{1}, \ldots, f_{l}\right)$ : $K^{n} \rightarrow K^{l}$, respectively, $U \rightarrow K^{l}$. Let $\Phi: K^{n} \rightarrow \mathbb{C}$ be a smooth function with compact support, i.e. $\Phi \in C_{0}^{\infty}$, with support in $U$ in the second case.

2.2. Log-principalization of ideals. We state the version of log-principalization of ideals that we will use in this paper, [7], see also [9], [22].

Theorem $1([7])$. Let $K=\mathbb{R}$ or $\mathbb{C}$ and let $U$ be an open submanifold of $K^{n}$. Let $f_{1}, \ldots, f_{l}$ be $K$-analytic functions on $U$ such that the ideal $\mathcal{I}_{\boldsymbol{f}}=\left(f_{1}, \ldots, f_{l}\right)$ is not trivial. Then there exists a log-principalization $h: X_{K} \rightarrow U$ of $\mathcal{I}_{\boldsymbol{f}}$, that is,

(1) $X_{K}$ is an $n$-dimensional $K$-analytic manifold, $h$ is a proper $K$-analytic map which can be chosen as a composition of a finite number of blow-ups in closed submanifolds, and which is an isomorphism outside of the common zero set $Z_{K}$ of $f_{1}, \ldots, f_{l}$;

(2) $h^{-1}\left(Z_{K}\right)=\cup_{i \in T} E_{i}$, where the $E_{i}$ are closed submanifolds of $X_{K}$ of codimension one, each equipped with a pair of positive integers $\left(N_{i}, v_{i}\right)$ satisfying the following. At every point $b$ of $X_{K}$ there exist local coordinates $\left(y_{1}, \ldots, y_{n}\right)$ on $X_{K}$ around $b$ such that, if $E_{1}, \ldots, E_{r}$ are the $E_{i}$ containing $b$, we have on some neighborhood of 
b that $E_{i}$ is given by $y_{i}=0$ for $i=1, \ldots, r$,

$$
h^{*}\left(\mathcal{I}_{\boldsymbol{f}}\right) \text { is generated by } \varepsilon(y) \prod_{i=1}^{r} y_{i}^{N_{i}}
$$

and

$$
h^{*}\left(d x_{1} \wedge \ldots \wedge d x_{n}\right)=\eta(y)\left(\prod_{i=1}^{r} y_{i}^{v_{i}-1}\right) d y_{1} \wedge \ldots \wedge d y_{n},
$$

where $\varepsilon(y), \eta(y)$ are units in the local ring of $X_{K}$ at $b$.

The $\left(N_{i}, v_{i}\right), i \in T$, are called the numerical data of $h$ for $\mathcal{I}_{\boldsymbol{f}}$.

2.3. Poles of local zeta functions. From now on we suppose that $\boldsymbol{f}^{-1}(0) \neq \emptyset$. To $\boldsymbol{f}$ and $\Phi$ as in 2.1 we associate the local zeta function $Z_{\Phi}(s, \boldsymbol{f}), s \in \mathbb{C}$ with $\operatorname{Re}(s)>0$, defined in the introduction.

Remark 1. (1) When $K=\mathbb{R}$ the zeta function $Z_{\Phi}(s, f)$ of the mapping $f$ is clearly equal to the zeta function $Z_{\Phi}\left(\frac{s}{2}, F\right)$ of the function $F:=\sum_{i=1}^{l} f_{i}^{2}$. In particular, it is known that $Z_{\Phi}(s, f)$ has a meromorphic continuation to the whole complex plane, and that its poles are negative rational numbers and of order at most $n$, see e.g. [13]. Also the list of candidate poles in Theorem 2 below for $K=\mathbb{R}$ can in fact be derived from the function case, see Proposition 1. But since the proof of Theorem 2 for $K=\mathbb{C}$ is also valid for $K=\mathbb{R}$ we prefer to state and prove it simultaneously for both fields.

(2) Over $K=\mathbb{C}$ the meromorphic continuation can be analogously reduced to the case of one real-analytic function. The point of Theorem 2 is the description of the candidate poles in terms of a principalization of the ideal.

Theorem 2. Let $\boldsymbol{f}$ and $\Phi$ be as in 2.1. Let $h: X_{K} \rightarrow U$ be a fixed log-principalization of the ideal $\mathcal{I}_{\boldsymbol{f}}=\left(f_{1}, \ldots, f_{l}\right)$, with numerical data $\left(N_{i}, v_{i}\right), i \in T$, for $\mathcal{I}_{\boldsymbol{f}}$. Then $Z_{\Phi}(s, \boldsymbol{f})$ has a meromorphic continuation to the whole complex plane $\mathbb{C}$ and the poles are contained in the union of

$$
-\frac{v_{i}}{N_{i}}-\frac{\mathbb{N}}{N_{i}}, i \in T
$$

Therefore the poles are negative rational numbers. Moreover their orders are at most equal to $n$.

Proof. We use all the notations concerning the log-principalization $h$ introduced in Theorem 1. Let $b \in X_{K}$ be a point, and $\left(\phi_{V}, V\right)$ a chart containing it. Let $E_{1}, \ldots, E_{r}$ denote the components of $h^{-1}\left(f^{-1}(0)\right)$ passing through $b$. We set $\boldsymbol{f}^{*}(y):=\boldsymbol{f}(h(y))$.

If $r=0$, i.e., $\boldsymbol{f}(h(b)) \neq 0$, then we can choose a small neighborhood $V_{b}$ of $b$ over which $\left|\boldsymbol{f}^{*}(y)\right|_{K}$ is positive and $\mathbb{R}$-analytic, and thus

$$
\left|\boldsymbol{f}^{*}(y)\right|_{K}^{s}=e^{s \ln \left|\boldsymbol{f}^{*}(y)\right|_{K}} \text { is } \mathbb{R} \text {-analytic in } y \in V_{b} \text { and holomorphic in } s \in \mathbb{C} .
$$

In addition,

$$
h^{*}\left(d x_{1} \wedge \ldots \wedge d x_{n}\right)=\eta(y)\left(d y_{1} \wedge \ldots \wedge d y_{n}\right),
$$

where $\eta(y)$ is a unit of the local ring of $X_{K}$ at $b$.

If $r \geq 1$, then we have in $V$ that

$$
f_{i}^{*}(y)=f_{i}(h(y))=g(y) \widetilde{f}_{i}(y), i=1, \ldots, l,
$$




$$
g(y)=\varepsilon(y) \prod_{i=1}^{r} y_{i}^{N_{i}}
$$

and

$$
h^{*}\left(d x_{1} \wedge \ldots \wedge d x_{n}\right)=\eta(y) \prod_{i=1}^{r} y_{i}^{v_{i}-1}\left(d y_{1} \wedge \ldots \wedge d y_{n}\right),
$$

where $\varepsilon(y)$ and $\eta(y)$ are units of the local ring of $X_{K}$ at $b$. Furthermore, there exists an index $i_{0}$ such that $\widetilde{f}_{i_{0}}(b) \neq 0$. Then

$$
\left|\boldsymbol{f}^{*}(y)\right|_{K}^{s}=|\varepsilon(y)|_{K}^{s}\left(\prod_{i=1}^{r}\left|y_{i}\right|_{K}^{N_{i}}\right)^{s}|\tilde{\boldsymbol{f}}(y)|_{K}^{s}
$$

where $\tilde{\boldsymbol{f}}(y):=\left(\widetilde{f}_{1}(y), \ldots, \widetilde{f}_{l}(y)\right)$, in $V$.

We can choose a small neighborhood $V_{b}$ of $b$ over which (2.3)-(2.5) are valid, and $|\varepsilon(y)|_{K},|\widetilde{\boldsymbol{f}}(y)|_{K},|\eta(y)|_{K}$ are $\mathbb{R}$-analytic. Then $|\varepsilon(y)|_{K}^{s},|\widetilde{\boldsymbol{f}}(y)|_{K}^{s}$ are $\mathbb{R}$-analytic in $y$ for any $s \in \mathbb{C}$, and holomorphic in $s \in \mathbb{C}$, for any $y \in V_{b}$.

Since $h^{-1}$ (support $\Phi$ ) is compact, we can take a finite covering of the form $\left\{V_{b}\right\}$ where the $V_{b}$ are homeomorphic under $\phi_{V}$ to the polydisc $P_{\epsilon}(0)$ in $K^{n}$ defined by $\left|y_{i}\right|_{K}<\epsilon$, with $\epsilon$ sufficiently small and for $1 \leq i \leq n$. By picking a smooth partition of the unity subordinate to $\left\{V_{b}\right\}$, and using the previous discussion,

$$
Z_{\Phi}(s, \boldsymbol{f})=\int_{X_{K} \backslash h^{-1}\left(\boldsymbol{f}^{-1}(0)\right)} \Phi^{*}(y)\left|\boldsymbol{f}^{*}(y)\right|_{K}^{s}\left|h^{*}\left(d x_{1} \wedge \ldots \wedge d x_{n}\right)\right|
$$

becomes a finite sum of integrals of the following two types:

$$
I(s):=\int_{K^{n}} \Psi(y)\left|\boldsymbol{f}^{*}(y)\right|_{K}^{s}|d y|,
$$

where $\Psi$ is a $C_{0}^{\infty}$ function with support contained in a polydisc $P_{\epsilon}(0)$ and $e^{s \ln \left|f^{*}(y)\right|_{K}}$ is $\mathbb{R}$-analytic for $y \in V_{b}$ and holomorphic in $s \in \mathbb{C}$, or

$$
J(s):=\int_{K^{n}} \Theta(y, s)\left(\prod_{i=1}^{r}\left|y_{i}\right|_{K}^{N_{i} s+v_{i}-1}\right)|d y|,
$$

where $\Theta(y, s)$ is a $C_{0}^{\infty}$ function with support contained in a polydisc $P_{\epsilon}(0)$, depending holomorphically on $s \in \mathbb{C}$. By using the Dominated Convergence Lemma, we have that (2.6) defines a holomorphic function on the complex plane. The meromorphic continuation and the description of the corresponding poles for integrals $J(s)$ is known, see, for instance, the proofs of Theorem 5.4.1 in [13] or Theorem 1.6 in [11].

We indicate know why also the list of candidate poles in Theorem 2 for $K=\mathbb{R}$ can be derived from the function case. We think this proposition has some independent interest. A note on terminology: we still use the term log-principalization starting with one function, i.e. with a principal ideal; usually one calls this an embedded resolution.

Proposition 1. Let $\boldsymbol{f}=\left(f_{1}, \ldots, f_{l}\right): U \rightarrow \mathbb{R}^{l}$ be an $\mathbb{R}$-analytic mapping on an open $U \subseteq \mathbb{R}^{n}$. Then $h: X_{\mathbb{R}} \rightarrow U$ is a log-principalization of the ideal $\mathcal{I}_{\boldsymbol{f}}=$ $\left(f_{1}, \ldots, f_{l}\right)$ if and only if it is a log-principalization of the function $F:=\sum_{i=1}^{l} f_{i}^{2}$. Moreover, when $\left(N_{i}, v_{i}\right), i \in T$, are the numerical data of $h$ for $\mathcal{I}_{\boldsymbol{f}}$, then $\left(2 N_{i}, v_{i}\right), i \in$ $T$, are the numerical data of $h$ for $F$. 
Proof. We suppose that $h$ is a log-principalization of the function $F$, with numerical data $\left(2 N_{i}, v_{i}\right), i \in T\left(2 N_{i}\right.$ will turn out to be even). We work in the local ring corresponding to some fixed point of $X_{\mathbb{R}}$; recall that this local ring is a unique factorization domain. Let $y_{1}, \ldots, y_{n}$ be coordinates at this point, i.e., a system of parameters of the local ring. We know that

$$
F^{*}(y)=\sum_{i=1}^{l}\left(f_{i}^{*}(y)\right)^{2}=\epsilon(y) \prod_{i=1}^{r} y_{i}^{2 N_{i}}
$$

where $\epsilon(y)$ is a unit and $r \leq n$. Let $g(y):=\operatorname{gcd}_{1 \leq i \leq l} f_{i}^{*}(y)$ and write $f_{i}^{*}(y)=$ $g(y) \tilde{f}_{i}(y)$.

We claim that $\sum_{i=1}^{l}\left(\widetilde{f}_{i}(y)\right)^{2}$ is a unit $u(y)$. Assuming the claim, we have that at least one of the $\widetilde{f}_{i}(y)$ is a unit. Hence

$$
h^{*} \mathcal{I}_{\boldsymbol{f}}=\left(f_{1}^{*}(y), \ldots, f_{l}^{*}(y)\right)=(g(y))
$$

and $\epsilon(y) \prod_{i=1}^{r} y_{i}^{2 N_{i}}=u(y)(g(y))^{2}$. So, up to a unit, $g(y)$ equals $\prod_{i=1}^{r} y_{i}^{N_{i}}$, and indeed $h$ is a $\log$-principalization of $\mathcal{I}_{\boldsymbol{f}}$.

We now prove the claim. We know that

$$
\epsilon(y) \prod_{i=1}^{r} y_{i}^{2 N_{i}}=(g(y))^{2}\left(\sum_{i=1}^{l}\left(\tilde{f}_{i}(y)\right)^{2}\right) .
$$

Since we have unique factorization, either this sum is a unit, or it is divisible by one of the irreducible elements of the left hand side, i.e., by $y_{1}, y_{2}, \ldots$ or $y_{r}$. Say $\sum_{i=1}^{l}\left(\widetilde{f}_{i}(y)\right)^{2}$ is divisible by $y_{1}$. We can always write this sum as

$$
\sum_{i=1}^{l}\left(\widetilde{f}_{i}\left(0, y_{2}, \ldots, y_{n}\right)\right)^{2}+y_{1}(\ldots) .
$$

Then divisibility by $y_{1}$ implies that $\sum_{i=1}^{l}\left(\widetilde{f}_{i}\left(0, y_{2}, \ldots, y_{n}\right)\right)^{2}=0$. Since we are working over $\mathbb{R}$ this can only happen if $\tilde{f}_{i}\left(0, y_{2}, \ldots, y_{n}\right)=0$ for all $i=1, \ldots, l$. But this is equivalent to $y_{1}$ dividing all $\widetilde{f}_{i}\left(y_{1}, y_{2}, \ldots, y_{n}\right)$, contradicting that the $\widetilde{f}_{i}(y)$ are relatively prime.

The other implication is quite straightforward.

Remark 2. We recall the description of the (local and global) log canonical threshold, see e.g. [6]-[16]-[19], in terms of a log-principalization. Let $K=\mathbb{R}$ or $\mathbb{C}$ and $U$ an open submanifold of $K^{n}$. Let $I_{f}=\left(f_{1}, \ldots, f_{l}\right)$ be a non-trivial ideal of $K$-analytic functions on $U$. Fix a $\log$-principalization $h: X_{K} \rightarrow U$ of $I_{f}$ as in Theorem 1.

(1) Let $p \in \boldsymbol{f}^{-1}(0)$. The $(K-) \log$ canonical threshold of $I_{\boldsymbol{f}}$ at $p$ is $c_{p}\left(I_{\boldsymbol{f}}\right)=$ $\min _{i \in T, p \in h\left(E_{i}\right)}\left\{\frac{v_{i}}{N_{i}}\right\}$.

(2) The (K-) log canonical threshold of $I_{\boldsymbol{f}}$ is $c\left(I_{\boldsymbol{f}}\right)=\min _{i \in T}\left\{\frac{v_{i}}{N_{i}}\right\}$.

Proposition 2. Let $\boldsymbol{f}$ and $\Phi$ be as in 2.1.

(1) Let $p \in \boldsymbol{f}^{-1}(0)$. If $\Phi$ is real and nonnegative with support in a small enough neighborhood of $p$ (in particular $\Phi(p)>0$ ), then $-c_{p}\left(\mathcal{I}_{\boldsymbol{f}}\right)$ is a pole of $Z_{\Phi}(s, \boldsymbol{f})$, more precisely its largest pole.

Fix a log-principalization of $\mathcal{I}_{\boldsymbol{f}}$ as in Theorem 1. 
(2) Say that $c\left(\mathcal{I}_{\boldsymbol{f}}\right)=\frac{v_{i}}{N_{i}}$ precisely for $i \in T_{\lambda}(\subset T)$. If $\Phi$ is real and nonnegative and its support intersects $h\left(\cup_{i \in T_{\lambda}} E_{i}\right)$, then $-c\left(\mathcal{I}_{\boldsymbol{f}}\right)$ is a pole of $Z_{\Phi}(s, \boldsymbol{f})$, more precisely its largest pole.

(3) Let $r\left(\mathcal{I}_{\boldsymbol{f}}\right)$ be the maximal number of $E_{i}, i \in T$, with $c\left(\mathcal{I}_{\boldsymbol{f}}\right)=\frac{v_{i}}{N_{i}}$, respectively $c_{p}\left(\mathcal{I}_{\boldsymbol{f}}\right)=\frac{v_{i}}{N_{i}}$, that have a nonempty intersection. Then $-c\left(\mathcal{I}_{\boldsymbol{f}}\right)$, respectively $-c_{p}\left(\mathcal{I}_{\boldsymbol{f}}\right)$, is a pole of order $r\left(\mathcal{I}_{\boldsymbol{f}}\right)$ of $Z_{\Phi}(s, \boldsymbol{f})$ when $\Phi$ is as above.

Proof. One uses the case of monomial integrals like in the case of one analytic function, see e.g. [1, Chap. II, $\S 7$ ]. The proof is a simple variation of the one given in [1, Chap. II, $\S 7$, Lemme 4] and [12, pp. 32-33] for the case $l=1$. For $K=\mathbb{R}$ one can alternatively use the case $l=1$ and Proposition 1 .

Note that this proposition gives an argument to see that these minima do not depend on the chosen log-principalization.

Corollary 1. Let $K$ be $\mathbb{R}$ or $\mathbb{C}$ as before, and $\boldsymbol{f}$ as in 2.1. Let $D$ be a compact subset of $K^{n}$. Then

(1) $|\boldsymbol{f}(x)|_{K}^{\delta}$ is locally integrable for $\delta>-c\left(\mathcal{I}_{\boldsymbol{f}}\right)$,

(2) $\operatorname{Vol}\left(\left\{\left.x \in D|| \boldsymbol{f}(x)\right|_{K} \leq \alpha\right\}\right) \leq \alpha^{c\left(\mathcal{I}_{\boldsymbol{f}}\right)-\epsilon} \int_{D}|\boldsymbol{f}(x)|_{K}^{-c\left(\mathcal{I}_{\boldsymbol{f}}\right)+\epsilon}|d x|$, for $\alpha>0$ and any small $\epsilon>0$.

Proof. The first part follows directly from Proposition 2. Then the second part follows via the Chebyshev inequality.

Such bounds on volumes have recently emerged as central to aspects of complex differential geometry, see [18] and references therein.

\section{Newton Polyhedra and Log-Principalizations}

We collect some results about Newton polyhedra and log-principalizations following [21] and the references therein. In this section we take again $K=\mathbb{R}$ or $K=\mathbb{C}$.

We set $\mathbb{R}_{+}:=\{x \in \mathbb{R} \mid x \geqslant 0\}$. Let $G$ be a nonempty subset of $\mathbb{N}^{n}$. The Newton polyhedron $\Gamma=\Gamma(G)$ associated to $G$ is the convex hull in $\mathbb{R}_{+}^{n}$ of the set $\cup_{m \in G}\left(m+\mathbb{R}_{+}^{n}\right)$. For instance classically one associates a Newton polyhedron (at the origin) to $g(x)=\sum_{m} c_{m} x^{m}\left(x=\left(x_{1}, \ldots, x_{n}\right), g(0)=0\right)$, being a nonconstant polynomial function over $K$ or a $K$-analytic function in a neighborhood of the origin, where $G=\operatorname{supp}(g):=\left\{m \in \mathbb{N}^{n} \mid c_{m} \neq 0\right\}$. Further we associate more generally a Newton polyhedron to an analytic mapping.

We fix a Newton polyhedron $\Gamma$ as above. Let $\langle\cdot, \cdot\rangle$ denote the usual inner product of $\mathbb{R}^{n}$, and identify the dual space of $\mathbb{R}^{n}$ with $\mathbb{R}^{n}$ itself by means of it.

For $a \in \mathbb{R}_{+}^{n}$, we define

$$
d(a, \Gamma)=d(a)=\min _{x \in \Gamma}\langle a, x\rangle,
$$

and the first meet locus $F(a)$ of $a$ as

$$
F(a):=\{x \in \Gamma \mid\langle a, x\rangle=d(a)\} .
$$

The first meet locus is a face of $\Gamma$. Moreover, if $a \neq 0, F(a)$ is a proper face of $\Gamma$.

We define an equivalence relation in $\mathbb{R}_{+}^{n}$ by taking $a \sim a^{\prime} \Leftrightarrow F(a)=F\left(a^{\prime}\right)$. The equivalence classes of $\sim$ are sets of the form

$$
\Delta_{\tau}=\left\{a \in \mathbb{R}_{+}^{n} \mid F(a)=\tau\right\},
$$


where $\tau$ is a face of $\Gamma$.

We recall that the cone strictly spanned by the vectors $a_{1}, \ldots, a_{r} \in \mathbb{R}_{+}^{n} \backslash\{0\}$ is the set $\Delta=\left\{\lambda_{1} a_{1}+\ldots+\lambda_{r} a_{r} \mid \lambda_{i} \in \mathbb{R}_{+}, \lambda_{i}>0\right\}$. If $a_{1}, \ldots, a_{r}$ are linearly independent over $\mathbb{R}, \Delta$ is called a simplicial cone. If $\left\{a_{1}, \ldots, a_{r}\right\}$ is a subset of a basis of the $\mathbb{Z}$-module $\mathbb{Z}^{n}$, we call $\Delta$ a simple cone.

A precise description of the geometry of the equivalence classes modulo $\sim$ is as follows. Each facet (i.e. a face of codimension one) $\gamma$ of $\Gamma$ has a unique vector $a(\gamma)=\left(a_{\gamma, 1}, \ldots, a_{\gamma, n}\right) \in \mathbb{N}^{n} \backslash\{0\}$, whose nonzero coordinates are relatively prime and which is perpendicular to $\gamma$. We denote by $\mathfrak{D}(\Gamma)$ the set of such vectors. The equivalence classes are rational cones of the form

$$
\Delta_{\tau}=\left\{\sum_{i=1}^{r} \lambda_{i} a\left(\gamma_{i}\right) \mid \lambda_{i} \in \mathbb{R}_{+}, \lambda_{i}>0\right\},
$$

where $\tau$ runs through the set of faces of $\Gamma$, and $\gamma_{i}, i=1, \ldots, r$ are the facets containing $\tau$. We note that $\Delta_{\tau}=\{0\}$ if and only if $\tau=\Gamma$. The family $\left\{\Delta_{\tau}\right\}_{\tau}$, with $\tau$ running over the proper faces of $\Gamma$, is a partition of $\mathbb{R}_{+}^{n} \backslash\{0\}$; we call this partition a polyhedral subdivision of $\mathbb{R}_{+}^{n}$ subordinated to $\Gamma$. We call $\left\{\bar{\Delta}_{\tau}\right\}_{\tau}$, the family formed by the topological closures of the $\Delta_{\tau}$, a fan subordinated to $\Gamma$.

Each cone $\Delta_{\tau}$ can be partitioned into a finite number of simplicial cones $\Delta_{\tau, i}$. In addition, the subdivision can be chosen such that each $\Delta_{\tau, i}$ is spanned by part of $\mathfrak{D}(\Gamma)$. Thus from the above considerations we have the following partition of $\mathbb{R}_{+}^{n} \backslash\{0\}:$

$$
\mathbb{R}_{+}^{n} \backslash\{0\}=\bigcup_{\tau}\left(\bigcup_{i=1}^{l_{\tau}} \Delta_{\tau, i}\right),
$$

where $\tau$ runs over the proper faces of $\Gamma$, and each $\Delta_{\tau, i}$ is a simplicial cone contained in $\Delta_{\tau}$.

By adding new rays, each simplicial cone can be partitioned further into a finite number of simple cones. In this way we obtain a simple polyhedral subdivision of $\mathbb{R}_{+}^{n}$ subordinated to $\Gamma$ and a simple fan subordinated to $\Gamma$ (see e.g. [14]).

3.1. The Newton polyhedron associated to an analytic mapping. Let $\boldsymbol{f}=$ $\left(f_{1}, \ldots, f_{l}\right), \boldsymbol{f}(0)=0$, be a nonconstant analytic mapping defined on a neighborhood $U \subset K^{n}$ of the origin. In [21] the authors associated to $f$ a Newton polyhedron $\Gamma(\boldsymbol{f}):=\Gamma\left(\cup_{i=1}^{l} \operatorname{supp}\left(f_{i}\right)\right)$, and a non-degeneracy condition to $\boldsymbol{f}$ and $\Gamma(\boldsymbol{f})$.

If $f_{i}(x)=\sum_{m} c_{m, i} x^{m}$, and $\tau$ is a face of $\Gamma(\boldsymbol{f})$, we set

$$
f_{i, \tau}(x):=\sum_{m \in \operatorname{supp}\left(f_{i}\right) \cap \tau} c_{m, i} x^{m} .
$$

Definition 1. Let $\boldsymbol{f}=\left(f_{1}, \ldots, f_{l}\right): U \longrightarrow K^{l}$ be a nonconstant analytic mapping satisfying $\boldsymbol{f}(0)=0$. The mapping $\boldsymbol{f}$ is called strongly non-degenerate at the origin with respect to $\Gamma(\boldsymbol{f})$, if for any compact face $\tau \subset \Gamma(\boldsymbol{f})$ and any $z \in$ $\left\{z \in\left(K^{\times}\right)^{n} \mid f_{1, \tau}(z)=\ldots=f_{l, \tau}(z)=0\right\}$ it satisfies $\operatorname{rank}\left[\frac{\partial f_{i, \tau}}{\partial x_{j}}(z)\right]=\min \{l, n\}$.

Remark 3. (1) The above notion of non-degeneracy agrees with the one given by Varchenko for the case $l=1$, see [20]. On the other hand, the previous notion does not agree with the non-degeneracy notion with respect to a collection of Newton 
polyhedra given by Khovanskii in [15]. We refer the reader to [21] for a further discussion about the mentioned non-degeneracy conditions.

(2) If we fix $l$ and $\Gamma$, then one can show, just as for the classical case $l=1$, that 'most' mappings $\boldsymbol{f}=\left(f_{1}, \ldots, f_{l}\right)$, with $\boldsymbol{f}(0)=0$ and Newton polyhedron $\Gamma(\boldsymbol{f})=\Gamma$, are strongly non-degenerate at the origin. One can easily generalize e.g. the proof of Lemme 1 in $[1$, p. 157]. However, just as for $l=1$, we should say that there are many interesting non-generic mappings.

(3) In [15], Khovanskii established the existence of an embedded resolution for a variety using a collection of Newton polyhedra, see also [1] for the case $l=1$. In [21], a log-principalization for an ideal with generators satisfying the above-mentioned notion of non-degeneracy and using one Newton polyhedron was established, see Proposition 3 below. This result agrees with Khovanskii's result only in the case $l=1$.

Remark 4. When $K=\mathbb{R}$ it is again natural to try to study the mapping $\boldsymbol{f}=$ $\left(f_{1}, \ldots, f_{l}\right)$ via the function $F:=\sum_{i=1}^{l} f_{i}^{2}$. It is not difficult to verify that $\Gamma(F)$ is the 'double' of $\Gamma(\boldsymbol{f})$, i.e. obtained from it after scaling by a factor 2 . Consider however the statements

(i) $F$ is non-degenerate at the origin with respect to $\Gamma(F)$, and

(ii) $\boldsymbol{f}$ is strongly non-degenerate at the origin with respect to $\Gamma(\boldsymbol{f})$.

It is easy to verify that (i) implies (ii), but in general the converse is not true. Consider for instance any strongly non-degenerate $\boldsymbol{f}$ for which $\left\{f_{1, \tau}=\ldots=f_{l, \tau}=\right.$ $0\} \cap\left(\mathbb{R}^{\times}\right)^{n} \neq \emptyset$ for some compact face $\tau$ of $\Gamma(\boldsymbol{f})$, e.g. $\boldsymbol{f}=\left(x^{2}-y^{3}, x^{2}-z^{3}\right)$.

In such cases the 'classical' embedded resolution of a non-degenerate function is not helpful, but one can use the log-principalization of a strongly non-degenerate mapping of Proposition 3 below.

\subsection{Log-principalizations.}

Proposition 3 ([21, Prop. 3.9]). Let $\boldsymbol{f}=\left(f_{1}, \ldots, f_{l}\right): U\left(\subset K^{n}\right) \longrightarrow K^{l}$ be a nonconstant analytic mapping, strongly non-degenerate at the origin with respect to $\Gamma(\boldsymbol{f})$. Let $\mathcal{F}_{\boldsymbol{f}}$ be a simple fan subordinated to $\Gamma(\boldsymbol{f})$. Let $Y_{K}$ be the toric manifold corresponding to $\mathcal{F}_{\boldsymbol{f}}$, and let

$$
\sigma_{0}: Y_{K} \longrightarrow U
$$

be the restriction of the corresponding toric map to the inverse image of $U$. Denote by $Z$ the set of common zeroes of $\mathcal{I}_{\boldsymbol{f}}=\left(f_{1}, \ldots, f_{l}\right)$ in $U \cap\left(K^{\times}\right)^{n}$. When $U$ is taken small enough, either $Z=\varnothing$ or it is a submanifold of codimension l. In this last case we have $l<n$ and we denote the closure of $Z$ in $Y_{K}$ by $Z_{Y}$.

(1) If $Z=\varnothing$ (or if $l=1$ ), the ideal $\sigma_{0}^{*}\left(\mathcal{I}_{\boldsymbol{f}}\right)$ is principal (and monomial) in a sufficiently small neighborhood of $\sigma_{0}^{-1}\{0\}$.

(2) If $Z \neq \varnothing$, we have that $Z_{Y}$ is a closed submanifold of $Y_{K}$, having normal crossings with the exceptional divisor of $\sigma_{0}$. Let $\sigma_{1}: X_{K} \longrightarrow Y_{K}$ be the blowing-up of $Y_{K}$ with center $Z_{Y}$, and let $\sigma=\sigma_{0} \circ \sigma_{1}: X_{K} \longrightarrow U$. Then the ideal $\sigma^{*}\left(\mathcal{I}_{\boldsymbol{f}}\right)$ is principal (and monomial) in a sufficiently small neighborhood of $\sigma^{-1}\{0\}$.

\section{Poles for local Zeta Functions and Newton Polyhedra}

Given $\xi=\left(\xi_{1}, \ldots, \xi_{n}\right) \in \mathbb{N}^{n} \backslash\{0\}$, we put $\sigma(\xi):=\xi_{1}+\ldots+\xi_{n}$ and $d(\xi)=$ $\min _{x \in \Gamma(\boldsymbol{f})}\langle\xi, x\rangle$ as before. We say that $\xi$ is a primitive vector, if $\operatorname{gcd}\left(\xi_{1}, \ldots, \xi_{n}\right)=1$. 
If $d(\xi) \neq 0$, we define

$$
\mathcal{P}(\xi)=\left\{-\frac{\sigma(\xi)+k}{d(\xi)} \mid k \in \mathbb{N}\right\}
$$

We also define

$$
\gamma_{0}(\boldsymbol{f})=\min _{\xi \in \mathfrak{D}(\Gamma(\boldsymbol{f}))}\left\{\frac{\sigma(\xi)}{d(\xi)}\right\} .
$$

Varchenko called $\gamma_{0}(\boldsymbol{f})$ the distance from the origin to $\Gamma(\boldsymbol{f})$. The number $\gamma_{0}(\boldsymbol{f})$ admits the following geometric interpretation. Let $\left(t_{0}, \ldots, t_{0}\right)$ be the intersection point of the diagonal $\left\{(t, \ldots, t) \in \mathbb{R}^{n} \mid t \in \mathbb{R}\right\}$ with the boundary of $\Gamma(\boldsymbol{f})$, then $\gamma_{0}(\boldsymbol{f})=1 / t_{0}$.

Let $\mathcal{F}_{\boldsymbol{f}}$ be a simple fan subordinated to $\Gamma(\boldsymbol{f})$. Then the set of generators of the cones in $\mathcal{F}_{\boldsymbol{f}}$, i.e. the skeleton of $\mathcal{F}_{\boldsymbol{f}}$, can be partitioned as $\Lambda_{\boldsymbol{f}} \cup \mathfrak{D}(\Gamma(\boldsymbol{f}))$, where $\Lambda_{f}$ is a finite set of primitive vectors, corresponding to the extra rays, induced by the subdivision into simple cones.

The numerical data of the log-principalizations constructed in Proposition 3 can be computed directly from the explicit expressions for the generators of $\sigma_{0}^{*}\left(\mathcal{I}_{\boldsymbol{f}}\right)$, $\sigma^{*}\left(\mathcal{I}_{\boldsymbol{f}}\right)$, and Lemme 8 in $[1$, p. 201]. The following theorem follows from the previous considerations by adapting the proof given by Varchenko for the case $l=1$ to arbitrary $l \geq 1$.

Theorem 3. Let $\boldsymbol{f}=\left(f_{1}, \ldots, f_{l}\right): U\left(\subset K^{n}\right) \longrightarrow K^{l}$, with $\boldsymbol{f}(0)=0$, be an analytic mapping, strongly non-degenerate at the origin with respect to $\Gamma(\boldsymbol{f})$. There exists a neighborhood $V(\subset U)$ of the origin such that, if $\Phi$ is a smooth function with support contained in $V$, then the following assertions hold.

(1) The function $Z_{\Phi}(s, \boldsymbol{f})$ is holomorphic on the complex half-plane $\operatorname{Re}(s)>$ $\max \left\{-\gamma_{0}(\boldsymbol{f}),-l\right\}$.

(2) The poles of $Z_{\Phi}(s, \boldsymbol{f})$ belong to the set $\cup_{\xi \in \mathfrak{D}(\Gamma(\boldsymbol{f}))} \mathcal{P}(\xi) \cup \cup_{\xi \in \Lambda_{\boldsymbol{f}}} \mathcal{P}(\xi) \cup(-(l+\mathbb{N}))$, where the last set may be discarded if $l \geq n$.

(3) If $\gamma_{0}(\boldsymbol{f})<l$, then $s=-\gamma_{0}(\boldsymbol{f})$ is a pole of $Z_{\Phi}(s, \boldsymbol{f})$ as a distribution on the space of smooth functions with compact support.

In the case $l=1$ and $K=\mathbb{R}$, Denef and Sargos proved in [5] that $\cup_{\xi \in \Lambda_{f}} \mathcal{P}(\xi)$ may be discarded from the list of candidate poles in Theorem 3. This is a strong and interesting result, yielding in general a much shorter list of candidate poles, that can moreover be read off immediately from $\Gamma(\boldsymbol{f})$.

In the next sections, we extend the Denef-Sargos result to arbitrary $l \geq 1$. Actually, we follow reasonably closely the approach of [5]. However, a number of extra difficulties pop up, for which a careful analysis is needed. In order to produce a readable text, we have to recall in the sequel the main ideas of [5]. Our goal will be to show the following result, for which we already indicate the starting point of its proof.

Theorem 4. Let $\boldsymbol{f}=\left(f_{1}, \ldots, f_{l}\right): U\left(\subset \mathbb{R}^{n}\right) \longrightarrow \mathbb{R}^{l}$, with $\boldsymbol{f}(0)=0$, be an analytic mapping, strongly non-degenerate at the origin with respect to $\Gamma(\boldsymbol{f})$. There exists a neighborhood $V(\subset U)$ of the origin such that, if $\Phi$ is a smooth function with support contained in $V$, then the following assertions hold.

(1) The poles of $Z_{\Phi}(s, \boldsymbol{f})$ belong to the set $\cup_{\xi \in \mathfrak{D}(\Gamma(\boldsymbol{f}))} \mathcal{P}(\xi) \cup(-(l+\mathbb{N}))$.

(2) Let $\rho$ be an integer satisfying $1 \leq \rho \leq n$, and let $s_{0}$ be a candidate pole of $Z_{\Phi}(s, \boldsymbol{f})$ with $s_{0} \notin-(l+\mathbb{N})$ (respectively $s_{0} \in-(l+\mathbb{N})$ ). A necessary condition 
for $s_{0}$ to be a pole of $Z_{\Phi}(s, \boldsymbol{f})$ of order $\rho$, is that there exists a face $\tau \subset \Gamma(\boldsymbol{f})$ of codimension $\rho$ (respectively of codimension $\rho-1$ ) such that $s_{0} \in \mathcal{P}(\xi)$ for any facet $F(\xi) \supset \tau$.

Proof. Define

$$
I_{\Phi}(s, \boldsymbol{f})=I(s, \boldsymbol{f}, \Phi)=\int_{\mathbb{R}_{+}^{n}} \Phi(x)|\boldsymbol{f}(x)|_{\mathbb{R}}^{s}|d x|, \operatorname{Re}(s)>0 .
$$

This integral defines an analytic function for $\operatorname{Re}(s)>0 ; I(s, \boldsymbol{f}, \Phi)$ and $Z_{\Phi}(s, \boldsymbol{f})$ are related by

$$
Z_{\Phi}(s, \boldsymbol{f})=\sum_{\theta \in\{-1,1\}^{n}} I(s, \boldsymbol{f}(\theta \cdot x), \Phi(\theta \cdot x)),
$$

where $\theta \cdot x=\left(\theta_{1} x_{1}, \ldots, \theta_{n} x_{n}\right)$. The result will follow from the meromorphic continuation of $I_{\Phi}(s, \boldsymbol{f})$, and the explicit description of its poles, cf. Theorem 6 .

\section{Monomial integrals and Decoupages}

From now on we take $K=\mathbb{R}$ and use all the notations introduced in the previous sections.

5.1. Some Monomial Integrals. We give some results about the meromorphic continuation of integrals attached to monomials that we will use later on. These results are easy variations of well-known results, see e.g. [1, Chap. II, $\S 7$, Lemme 3], [5, Lemme 3.1], [8, Chap. I, Sect. 3.2], and [11, pp. 101-102].

Lemma 1. Let $\Omega$ be an open neighborhood of the origin in $\mathbb{R}^{k} \times \mathbb{R}$ and let $g$ : $\Omega \rightarrow \mathbb{R}$ be an $\mathbb{R}$-analytic function. Let $\phi$ be a smooth function with support in $\Omega$ and containing the origin. Take $a=\left(a_{1}, \ldots, a_{k+1}\right) \in\left(\mathbb{R}_{+} \backslash\{0\}\right)^{k+1}$ such that $\left[0, a_{1}\right] \times \cdots \times\left[0, a_{k+1}\right]$ is contained in the support of $\phi$. Assume that $g(y, z)>0$ for $(y, z)$ in the support of $\phi$, and define

$J(s)=\int_{0}^{a_{1}} \cdots \int_{0}^{a_{k+1}}\left(\prod_{j=1}^{r} y_{j}^{s m_{j}+\gamma_{j}-1}\right) z^{s+l-1} g(y, z)^{s} \phi(y, z)|d y \wedge d z|, \quad \operatorname{Re}(s)>0$, with $1 \leq r \leq k$ and $m_{j}, \gamma_{j} \in \mathbb{N} \backslash\{0\}$ for $j=1, \ldots, r$, and $l \in \mathbb{N} \backslash\{0\}$. Then the following assertions hold:

(1) $J(s)$ is convergent and defines a holomorphic function on

$$
\operatorname{Re}(s)>\max \left\{-l,-\gamma_{1} / m_{1}, \ldots,-\gamma_{r} / m_{r}\right\} ;
$$

(2) $J(s)$ admits a meromorphic continuation to the whole complex plane, with poles of order at most $k+1$. Furthermore, the poles belong to

$$
\bigcup_{1 \leq i \leq r}\left(-\frac{\gamma_{i}+\mathbb{N}}{m_{i}}\right) \cup(-(l+\mathbb{N})) .
$$

(3) Let $\rho$ be a positive integer and let $s_{0}$ be a candidate pole of $J(s)$ with $s_{0} \notin$ $-(l+\mathbb{N})\left(\right.$ resp. $\left.s_{0} \in-(l+\mathbb{N})\right)$. A necessary condition for $s_{0}$ to be a pole of $J(s)$ of order $\rho$, is that

$$
\operatorname{Card}\left\{i \mid s_{0} \in \frac{-\left(\gamma_{i}+\mathbb{N}\right)}{m_{i}}\right\} \geq \rho(\text { resp. } \geq \rho-1) .
$$


Corollary 2. Let $r, k$ and $l$ be natural numbers such that $1 \leq r \leq k$ and $k+1-$ $r-l \geq 0$. Let $U$ be a neighborhood of the origin of $\mathbb{R}^{l}$. Let $\theta$ be a smooth function with compact support contained in $[0,1]^{r} \times U \times[0,1]^{k+1-r-l}$. Set

$$
J_{1}(s):=\int_{[0,1]^{r} \times U \times[0,1]^{k+1-r-l}} \prod_{j=1}^{r} y_{j}^{s m_{j}+\gamma_{j}-1}\left(\sum_{i=r+1}^{r+l} y_{i}^{2}\right)^{s / 2} \theta(y)|d y|, \operatorname{Re}(s)>0 .
$$

Then $J_{1}(s)$ has a meromorphic continuation to $\mathbb{C}$ and its poles satisfy all the conclusions in Lemma 1.

Proof. The result follows from the previous lemma by using hyper-spherical coordinates.

5.2. Decoupages in $\mathbb{R}^{n}$ and compatible mappings. Let $\boldsymbol{f}=\left(f_{1}, \ldots, f_{l}\right): U(\subset$ $\left.\mathbb{R}^{n}\right) \longrightarrow \mathbb{R}^{l}$ be an analytic mapping, strongly non-degenerate at the origin with respect to $\Gamma(\boldsymbol{f})$.

We fix a simplicial fan $\mathcal{F}_{\boldsymbol{f}}$ subordinated to $\Gamma(\boldsymbol{f})$, and assume that the cones of $\mathcal{F}_{f}$ of dimension $n$ are $\bar{\Delta}_{1}, \ldots, \bar{\Delta}_{m}$.

Definition 2. Let $X \subseteq \mathbb{R}^{n}$ be a measurable set in the sense of Lebesgue. A decoupage of $X$ is a finite family $\mathcal{D}=\left\{D_{1}, \ldots, D_{m}\right\}$ of measurable sets satisfying

(1) $D_{i} \cap D_{j}$ has measure zero for $i \neq j$, and

(2) $X \backslash\left(\cup_{i=1}^{m} D_{i}\right)$ and $\left(\cup_{i=1}^{m} D_{i}\right) \backslash X$ have measure zero.

Define

$$
\mathcal{L}:(0,1]^{n} \rightarrow \mathbb{R}_{+}^{n}:\left(x_{1}, \ldots, x_{n}\right) \rightarrow\left(-\ln x_{1}, \ldots,-\ln x_{n}\right) .
$$

Then $\mathcal{L}$ is an $\mathbb{R}$-analytic isomorphism, and $\left\{\mathcal{L}^{-1}\left(\bar{\Delta}_{j}\right)\right\}_{1 \leq j \leq m}$ is a decoupage of $[0,1]^{n}$. In addition, for any smooth function $\phi(x)$,

$$
\int_{[0,1]^{n}} \phi(x)|d x|=\sum_{j=1}^{m} \int_{\mathcal{L}^{-1}\left(\bar{\Delta}_{j}\right)} \phi(x)|d x| .
$$

In the sequel we will use a decoupage of the domain of integration to give a short list of candidate poles for local zeta functions. It may happen that a mapping $\left(f_{1, \tau}(x), \ldots, f_{l, \tau}(x)\right)$, restricted to certain components of the boundary of a decoupage, has singularities. These cases will turn out be to annoying, but they are rare and they can be removed by using homotheties, cf. Lemma 3.

Take vectors $a_{1}, \ldots, a_{n} \in \mathbb{N}^{n}$ that are linearly independent over $\mathbb{R}$, and denote

$$
\bar{\Delta}:=\left\{\sum_{i=1}^{n} \lambda_{i} a_{i} \mid \lambda_{i} \in \mathbb{R}_{+}\right\}
$$

for the closed cone spanned by them. For $K \subset\{1, \ldots, n\}$, set

$$
\Delta_{K}:=\left\{\sum_{i \in K} \lambda_{i} a_{i} \mid \lambda_{i} \in \mathbb{R}_{+}, \lambda_{i}>0\right\}
$$

and $S_{K}:=\mathcal{L}^{-1}\left(\Delta_{K}\right)$, with the convention that $S_{K}=\{(1, \ldots, 1)\}$ if $K=\emptyset$. We will call $S_{K}$ a sector.

Definition 3. We say that $\boldsymbol{f}$ is compatible with the sector $S_{K}$ if the following condition is satisfied. For each partition $\{I, J, K\}$ of $\{1, \ldots, n\}$, with $I \neq \emptyset, J \neq \emptyset$, and such that the face $\tau=\cap_{i \in I} F\left(a_{i}\right)$ is compact and non-empty, the restriction 
to $S_{K}$ of the map $\boldsymbol{f}_{\tau}:=\left(f_{1, \tau}, \ldots, f_{l, \tau}\right)$ does not admit $(0, \ldots, 0) \in \mathbb{R}^{l}$ as a critical value.

We say that $\boldsymbol{f}$ is compatible with $\mathcal{L}^{-1}(\bar{\Delta})$, if $\boldsymbol{f}$ is compatible with the sector $S_{K}$ for any $K \subset\{1, \ldots, n\}$.

We say that $\boldsymbol{f}$ is compatible with the decoupage $\mathcal{L}^{-1}\left(\mathcal{F}_{f}\right)=\left\{\mathcal{L}^{-1}(\bar{\Delta}) \mid \bar{\Delta} \in \mathcal{F}_{f}\right.$ with $\operatorname{dim} \bar{\Delta}=n\}$ (or compatible with $\mathcal{F}_{\boldsymbol{f}}$ ), if $\boldsymbol{f}$ is compatible with $\mathcal{L}^{-1}(\bar{\Delta})$, for any $n$-dimensional cone $\bar{\Delta}$ in $\mathcal{F}_{\boldsymbol{f}}$.

Remark 5. Note that the condition 'the restriction to $S_{K}$ of $\boldsymbol{f}_{\tau}$ does not admit $(0, \ldots, 0) \in \mathbb{R}^{l}$ as a critical value' is equivalent to the two following conditions:

(1) if $\operatorname{Card}(K) \geq l$, then $\operatorname{rank}\left[\frac{\partial f_{i, \tau}}{\partial x_{j}}(z)\right]=l$, for any $z \in\left\{z \in S_{K} \mid f_{1, \tau}(z)=\cdots=\right.$ $\left.f_{l, \tau}(z)=0\right\}$, and

(2) if $0 \leq \operatorname{Card}(K) \leq l-1$, then for any $z_{0} \in S_{K}$, there exists an index $i_{0}=i_{0}\left(z_{0}\right)$, such that $f_{i_{0}, \tau}\left(z_{0}\right) \neq 0$.

Remark 6. Set $n=l=2$. Since any partition $\{I, J, K\}$ of $\{1,2\}$, with $I \neq \emptyset$ and $J \neq \emptyset$, requires that $K=\emptyset$ and that $\operatorname{Card}(I)=1$, any $\tau$ in Definition 3 is a facet of $\Gamma(\boldsymbol{f})$. Therefore $\boldsymbol{f}$ is compatible with a simplicial fan $\mathcal{F}_{\boldsymbol{f}}$ if and only if $f_{1, \gamma}(1,1) \neq 0$ or $f_{2, \gamma}(1,1) \neq 0$, for any compact facet $\gamma$ of $\Gamma(\boldsymbol{f})$.

Example 1. Set $\boldsymbol{f}=\left(x_{2}^{a}-x_{1}^{b}, x_{1}^{c}-x_{2}^{d}\right)$, with $a \leq d$ and $b \leq c$. Then $\boldsymbol{f}$ is not compatible with any simplicial fan $\mathcal{F}_{\boldsymbol{f}}$, since $f_{i, \gamma}(1,1)=0(i=1,2)$ for the facet $\gamma$ containing $(0, a)$ and $(b, 0)$. Set $T_{(3,2)}:\left(\mathbb{R}_{+} \backslash\{0\}\right)^{2} \rightarrow\left(\mathbb{R}_{+} \backslash\{0\}\right)^{2} ;\left(x_{1}, x_{2}\right) \mapsto$ $\left(3 x_{1}, 2 x_{2}\right)$. Then $\boldsymbol{f} \circ T_{(3,2)}$ is compatible with every simplicial fan $\mathcal{F}_{\boldsymbol{f}}$.

Example 2. Set $\mathbf{g}=\left(x_{2}^{a}+x_{1}^{b}, x_{1}^{c}\right)$, with $c \leq b$. Then $\mathbf{g}$ is compatible with every simplicial fan $\mathcal{F}_{\boldsymbol{f}}$.

5.3. Compatibility and homotheties. Subsequently we will use only the part of the following lemma, stating that the sets $T_{l, k^{\prime}}$ have measure zero. But we think it is natural to state and prove it as below.

Lemma 2. Assume $l \leq n$. Let $W_{0} \times W_{1}$ be an open neighborhood of $(0,0) \in \mathbb{R}^{n} \times \mathbb{R}^{n}$, and $\mathbf{h}: W_{0} \times W_{1} \rightarrow \mathbb{R}^{l}:(t, z) \mapsto \mathbf{h}(t, z)=\left(h_{1}(t, z), \ldots, h_{l}(t, z)\right)$ an analytic mapping such that $(0, \ldots, 0) \in \mathbb{R}^{l}$ is not a critical value of $\mathbf{h}$. Set

$$
\begin{aligned}
& M:=\left\{(t, z) \in W_{0} \times W_{1} \mid h_{1}(t, z)=\cdots=h_{l}(t, z)=0\right\}, \\
& \pi: M \rightarrow \mathbb{R}^{n}:(t, z) \mapsto t, \text { and } \\
& T_{l^{\prime}, k^{\prime}}:=\left\{\begin{array}{l}
t \in W_{0} \mid \mathbf{h}(t, z)=0 \text { for some } z \in W_{1} \text { and } \\
\operatorname{rank}\left[\frac{\partial h_{i}}{\partial z_{k}}(t, z)\right]_{\substack{1 \leq i \leq l^{\prime} \\
k^{\prime} \leq k \leq n}}<\min \left\{l^{\prime}, n-k^{\prime}+1\right\}
\end{array}\right\}
\end{aligned}
$$

for $1 \leq l^{\prime} \leq l$ and $1 \leq k^{\prime} \leq n$. Then $T_{l^{\prime}, k^{\prime}}$ has measure zero, and the set of critical values of $\pi$ is the union of the sets $T_{l^{\prime}, k^{\prime}}$ and of the similar sets obtained by permuting the indices.

Proof. We establish the result by showing explicitly that the set of critical values of $\pi$ restricted to a neighborhood $M_{(a, b)}$ of point $(a, b)$ in $M$ has the form $T_{l^{\prime}, k^{\prime}}$. Then by Sard's lemma $T_{l^{\prime}, k^{\prime}}$ has measure zero, for any $l^{\prime}, k^{\prime}$. This explicit description is achieved as follows: given $(a, b) \in M$, by the implicit function theorem, we can solve the system $\left\{h_{1}(t, z)=\cdots=h_{l}(t, z)=0\right\}$ locally around $(a, b)$, for $l$ variables 
in terms of the others. Depending on the selection made upon the set of variables several cases occur.

Case 1. All the depending variables are taken from the first $n$ variables. After renaming the variables if necessary, we assume that

$$
\operatorname{det}\left[\frac{\partial h_{i}}{\partial t_{j}}((a, b))\right]_{\substack{1 \leq i \leq l \\ 1 \leq j \leq l}} \neq 0
$$

Set $t=\left(t^{\prime}, t^{\prime \prime}\right)$, where $t^{\prime}=\left(t_{1}, \ldots, t_{l}\right)$ and $t^{\prime \prime}=\left(t_{l+1}, \ldots, t_{n}\right)$. We also set $(a, b)=\left(a^{\prime}, a^{\prime \prime}, b\right) \in\left(\mathbb{R}^{l} \times \mathbb{R}^{n-l} \times \mathbb{R}^{n}\right) \cap M$. Then there exist an open set $G \subset$ $\mathbb{R}^{2 n-l}$, containing $\left(a^{\prime \prime}, b\right)$, and a unique analytic function $\mathbf{g}: G \rightarrow \mathbb{R}^{l}$, such that $\mathbf{g}\left(a^{\prime \prime}, b\right)=a^{\prime}$ and $\mathbf{h}\left(\mathbf{g}\left(t^{\prime \prime}, z\right), t^{\prime \prime}, z\right)=0$ for any $\left(t^{\prime \prime}, z\right) \in G$.

Set $M_{(a, b)}=\left\{\left(t^{\prime}, t^{\prime \prime}, z\right) \in \mathbf{g}(G) \times G \mid t_{1}=g_{1}\left(t^{\prime \prime}, z\right), \ldots, t_{l}=g_{l}\left(t^{\prime \prime}, z\right)\right\} \subseteq M$. Then

$$
\begin{aligned}
& \left.\pi\right|_{M_{(a, b)}}: M_{(a, b)} \quad \rightarrow \quad \mathbb{R}^{n} \\
& \left(t^{\prime \prime}, z\right) \mapsto\left(g_{1}\left(t^{\prime \prime}, z\right), \ldots, g_{l}\left(t^{\prime \prime}, z\right), t^{\prime \prime}\right),
\end{aligned}
$$

and the Jacobian matrix of $\left.\pi\right|_{M_{(a, b)}}$ is

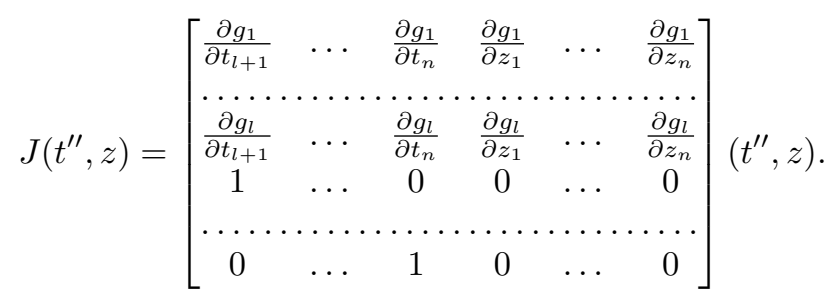

The point $\left(t^{\prime}, t^{\prime \prime}, z\right)$ is a critical point for $\pi$ if $\operatorname{rank} J\left(t^{\prime \prime}, z\right)<n$; this means that $\operatorname{rank}\left[\frac{\partial g_{i}}{\partial z_{k}}\left(t^{\prime \prime}, z\right)\right]_{\substack{1 \leq i \leq l \\ 1 \leq k \leq n}}<l$. Since $\sum_{j=1}^{l}\left(\frac{\partial h_{i}}{\partial t_{j}}\right)\left(\frac{\partial g_{j}}{\partial z_{k}}\right)=\frac{\partial h_{i}}{\partial z_{k}}$ and the matrix $\left[\frac{\partial h_{i}}{\partial t_{j}}\right]_{\substack{1 \leq i \leq l \\ 1 \leq j \leq l}}$ has maximal rank around $(a, b)$, this last condition is equivalent to

$$
\operatorname{rank}\left[\frac{\partial h_{i}}{\partial z_{k}}\left(\left(t^{\prime}, t^{\prime \prime}, z\right)\right)\right]_{\substack{1 \leq i \leq l \\ 1 \leq k \leq n}}<l .
$$

Hence $T_{l, 1}$ is the set of critical values of $\left.\pi\right|_{M_{(a, b)}}$.

Case 2. Some of the depending variables are taken from the first $n$ variables ( $t$-variables) and the rest are taken from the last $n$ variables ( $z$-variables). After renaming the variables if necessary, we assume that

$$
\left.\operatorname{det}\left[\frac{\partial h_{i}}{\partial t_{j}}((a, b))\right]_{\substack{1 \leq i \leq l \\ n-l^{\prime}+1 \leq j \leq n}}\left[\frac{\partial h_{i}}{\partial z_{j}}((a, b))\right]_{\substack{1 \leq i \leq l \\ 1 \leq j \leq l^{\prime \prime}}}\right] \neq 0,
$$

with $l=l^{\prime}+l^{\prime \prime}$. Put $t^{\prime}=\left(t_{1}, \ldots, t_{n-l^{\prime}}\right), t^{\prime \prime}=\left(t_{n-l^{\prime}+1}, \ldots, t_{n}\right), z^{\prime}=\left(z_{1}, \ldots, z_{l^{\prime \prime}}\right)$ and $z^{\prime \prime}=\left(z_{l^{\prime \prime}+1}, \ldots, z_{n}\right)$, hence $t=\left(t^{\prime}, t^{\prime \prime}\right)$ and $z=\left(z^{\prime}, z^{\prime \prime}\right)$. Then the depending variables are $t^{\prime \prime}$ and $z^{\prime}$. Consider a point $(a, b)=\left(a^{\prime}, a^{\prime \prime}, b^{\prime}, b^{\prime \prime}\right) \in\left(\mathbb{R}^{n-l^{\prime}} \times \mathbb{R}^{l^{\prime}} \times\right.$ $\left.\mathbb{R}^{l^{\prime \prime}} \times \mathbb{R}^{n-l^{\prime \prime}}\right) \cap M$. Then there exist an open set $Q \subset \mathbb{R}^{2 n-l}$, containing $\left(a^{\prime}, b^{\prime \prime}\right)$, 
and a unique analytic function $\mathbf{q}: Q \rightarrow \mathbb{R}^{l}$ such that $\mathbf{q}\left(a^{\prime}, b^{\prime \prime}\right)=\left(a^{\prime \prime}, b^{\prime}\right)$ and $\mathbf{h}\left(t^{\prime}, \mathbf{q}\left(t^{\prime}, z^{\prime \prime}\right), z^{\prime \prime}\right)=0$, for any $\left(t^{\prime}, z^{\prime \prime}\right) \in Q$. Set

$$
\begin{gathered}
M_{(a, b)}:= \\
\left\{\begin{array}{l}
\left(t^{\prime}, t^{\prime \prime}, z^{\prime}, z^{\prime \prime}\right) \in W_{0} \times W_{1} \mid\left(t^{\prime}, z^{\prime \prime}\right) \in Q,\left(t^{\prime \prime}, z^{\prime}\right) \in \mathbf{q}(Q) \text { and } \\
t_{n-l^{\prime}+1}=q_{1}\left(t^{\prime}, z^{\prime \prime}\right), \ldots, t_{n}=q_{l^{\prime}}\left(t^{\prime}, z^{\prime \prime}\right), z_{1}=q_{l^{\prime}+1}\left(t^{\prime}, z^{\prime \prime}\right), \ldots, z_{l^{\prime \prime}}=q_{l}\left(t^{\prime}, z^{\prime \prime}\right)
\end{array}\right\} .
\end{gathered}
$$

Then

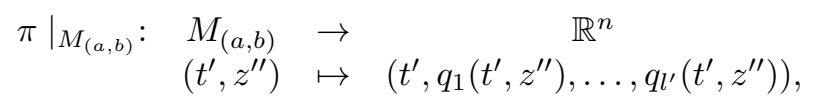

and the corresponding Jacobian matrix is

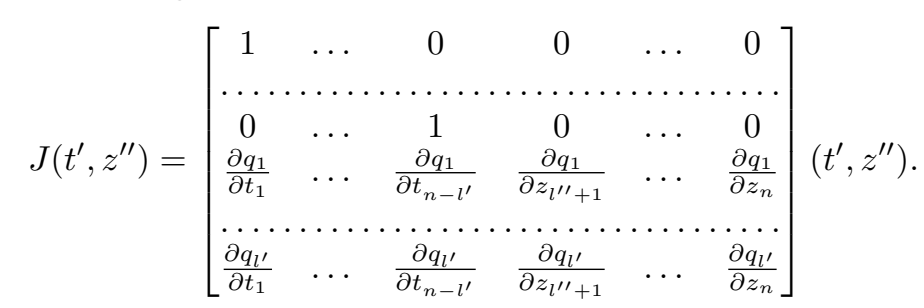

The point $\left(t^{\prime}, t^{\prime \prime}, z^{\prime}, z^{\prime \prime}\right)$ is a critical point for $\left.\pi\right|_{M_{(a, b)}}$ if $\operatorname{rankJ}\left(t^{\prime}, z^{\prime \prime}\right)<n$, that is,

$$
\operatorname{rank}\left[\frac{\partial q_{i}}{\partial z_{k}}\left(t^{\prime}, z^{\prime \prime}\right)\right]_{\substack{1 \leq i \leq l^{\prime} \\ k^{\prime} \leq k \leq n}}<l^{\prime}=\min \left\{l^{\prime}, n-l^{\prime \prime}\right\}
$$

where $k^{\prime}=l^{\prime \prime}+1$. As above, this is equivalent with $\operatorname{rank}\left[\frac{\partial h_{i}}{\partial z_{k}}\left(t^{\prime}, t^{\prime \prime}, z^{\prime}, z^{\prime \prime}\right)\right]_{\substack{1 \leq i \leq l^{\prime} \\ k^{\prime} \leq k \leq n}}<$ $l^{\prime}$, hence $T_{l^{\prime}, k^{\prime}}$ is the set of critical values of $\left.\pi\right|_{M_{(a, b)}}$.

Given $b \in\left(\mathbb{R}_{+} \backslash\{0\}\right)^{n}$, we define

$$
\begin{array}{cccc}
T_{b}: & \left(\mathbb{R}^{\times}\right)^{n} & \rightarrow & \left(\mathbb{R}^{\times}\right)^{n} \\
& \left(x_{1}, \ldots, x_{n}\right) & \mapsto & \left(b_{1} x_{1}, \ldots, b_{n} x_{n}\right) .
\end{array}
$$

Lemma 3. Let $\boldsymbol{f}$ be a strongly non-degenerate analytic mapping at the origin with respect to $\Gamma(\boldsymbol{f})$. Let $\mathcal{L}^{-1}\left(\mathcal{F}_{\boldsymbol{f}}\right)$ be a decoupage induced by a simplicial fan subordinated to $\Gamma(\boldsymbol{f})$. Then for almost every $b \in\left(\mathbb{R}_{+} \backslash\{0\}\right)^{n}$, the mapping $\boldsymbol{f} \circ T_{b}$ is compatible with $\mathcal{L}^{-1}\left(\mathcal{F}_{\boldsymbol{f}}\right)$.

Proof. We use all the notation introduced in Section 5.2 and Definition 3. Set $\bar{\Delta}=\left\{\sum_{i=1}^{n} \lambda_{i} a_{i} \mid \lambda_{i} \in \mathbb{R}_{+}\right\}$a cone in $\mathcal{F}_{\boldsymbol{f}},\{I, J, K\}$ a partition of $\{1, \ldots, n\}$ with $I \neq \emptyset$ and $J \neq \emptyset$, and $\Delta_{K}=\left\{\sum_{i \in K} \lambda_{i} a_{i} \mid \lambda_{i} \in \mathbb{R}_{+}, \lambda_{i}>0\right\}$ for $K \subset\{1, \ldots, n\}$. Assume $\tau=\cap_{i \in I} F\left(a_{i}\right)$ is non-empty and compact.

Case $(\operatorname{Card}(K) \geq l)$. Note that in this case necessarily $l<n($ since $\operatorname{Card}(K)<$ $n)$. We set $\psi:=\mathcal{L}^{-1} \circ \theta$ where

$$
\mathbb{R}^{n} \stackrel{\theta}{\longrightarrow} \mathbb{R}^{n} \stackrel{\mathcal{L}^{-1}}{\longrightarrow}\left(\mathbb{R}_{+} \backslash\{0\}\right)^{n},
$$

$\theta(z)=\sum_{i=1}^{n} z_{i} a_{i}$, and the extension of $\mathcal{L}$ to $\left(\mathbb{R}_{+} \backslash\{0\}\right)^{n}$ is denoted again by $\mathcal{L}$. We also set

$$
U=\left\{z \in \mathbb{R}^{n} \mid z_{j}=0 \text { if } j \notin K, \text { and } z_{j}>0 \text { if } j \in K\right\}
$$


and

$$
N=\left\{\begin{array}{l}
b \in\left(\mathbb{R}_{+} \backslash\{0\}\right)^{n} \mid\left(\boldsymbol{f}_{\tau} \circ T_{b} \circ \psi\right)(z)=0 \text { and } \\
\operatorname{rank}\left[\frac{\partial\left(f_{i, \tau} \circ T_{b} \circ \psi\right)}{\partial z_{k}}(z)\right]_{\substack{1 \leq i \leq l \\
k \in \bar{K}}}<\min \{l, \operatorname{Card}(K)\}=l, \text { for some } z \in U
\end{array}\right\} .
$$

To establish this case, we show that $N$ has measure zero. We may assume that $K=\left\{k^{\prime}, k^{\prime}+1, \ldots, n\right\}$, by renaming the coordinates, with $k^{\prime} \leq n-l+1$.

Note that for any $b \in\left(\mathbb{R}_{+} \backslash\{0\}\right)^{n}$ there exists a $t \in \mathbb{R}^{n}$ such that $b=\psi(t)$, and

$$
\begin{aligned}
\left(\boldsymbol{f}_{\tau} \circ T_{b} \circ \psi\right)(z) & =\boldsymbol{f}_{\tau}\left(T_{\psi(t)} \circ \psi\right)(z)=\boldsymbol{f}_{\tau}\left(\psi_{1}(t) \psi_{1}(z), \ldots, \psi_{n}(t) \psi_{n}(z)\right) \\
& =\boldsymbol{f}_{\tau}\left(\psi_{1}(t+z), \ldots, \psi_{n}(t+z)\right)=\left(\boldsymbol{f}_{\tau} \circ \psi\right)(t+z) .
\end{aligned}
$$

Since $\boldsymbol{f}$ is strongly non-degenerate at the origin with respect to $\Gamma(\boldsymbol{f})$ and $\psi$ is an analytic isomorphism over $\left(\mathbb{R}^{\times}\right)^{n}$, by taking $l^{\prime}=l$ in Lemma 2 we conclude that

$$
N^{\prime}=\left\{\begin{array}{l}
t \in \mathbb{R}^{n} \mid\left(\boldsymbol{f}_{\tau} \circ \psi\right)(t+z)=0 \quad \text { and } \\
\operatorname{rank}\left[\frac{\partial\left(f_{i, \tau} \circ \psi\right)}{\partial z_{k}}(t+z)\right]_{\substack{1 \leq i \leq l \\
k^{\prime} \leq k \leq n}}<l, \text { for some } z \in U
\end{array}\right\}
$$

has measure zero. Since $\psi$ is an analytic isomorphism, $N$ has measure zero too.

Case $(0 \leq \operatorname{Card}(K) \leq l-1)$. We show that the set of the $b$ 's such that $\left(\boldsymbol{f}_{\tau} \circ T_{b}\right)\left(z_{0}\right)=0$ for $z_{0} \in S_{K}$ has measure zero. This is equivalent to show that $Y_{z_{0}}=\left\{t \in \mathbb{R}^{n} \mid\left(\boldsymbol{f}_{\tau} \circ \psi\right)\left(t+z_{0}\right)=0\right\}$, for a $z_{0} \in U$, has measure zero. Finally $Y_{z_{0}}$ has measure zero, because it is a proper $\mathbb{R}$-analytic subset of $\mathbb{R}^{n}$.

\section{Short List OF CANDIDATE POLES}

6.1. Integral restricted to a sector. Let $a_{1}, \ldots, a_{n} \in \mathbb{N}^{n}$, determining linearly independent vectors of $\mathbb{R}^{n}$, and $\bar{\Delta}_{\tau}=\left\{\lambda_{1} a_{1}+\cdots+\lambda_{n} a_{n} \mid \lambda_{i} \in \mathbb{R}_{+}\right\}$a closed cone in a fixed simplicial fan $\mathcal{F}_{\boldsymbol{f}}$ subordinated to $\Gamma(\boldsymbol{f})$, such that $F(a)=\tau$ for any $a \in \Delta_{\tau}$. Set

$$
I_{\bar{\Delta}_{\tau}}(s):=I_{\bar{\Delta}_{\tau}}(s, \boldsymbol{f}, \Phi)=\int_{\mathcal{L}^{-1}\left(\bar{\Delta}_{\tau}\right)} \Phi(x)|\boldsymbol{f}(x)|_{\mathbb{R}}^{s}|d x|,
$$

for $\operatorname{Re}(s)>0$, where $\Phi$ is a smooth function with compact support, contained in a neighborhood of the origin.

6.1.1. The change of variables for a cone. Set $x \in(0,1]^{n}$ such that $\mathcal{L}(x) \in \bar{\Delta}_{\tau}$. Then

$\mathcal{L}(x)=\left(-\ln x_{1}, \ldots,-\ln x_{n}\right)=\lambda_{1} a_{1}+\cdots+\lambda_{n} a_{n}=\left(-\ln y_{1}\right) a_{1}+\cdots+\left(-\ln y_{n}\right) a_{n}$, for some unique $y=\left(y_{1}, \ldots, y_{n}\right) \in(0,1]^{n}$ satisfying $\mathcal{L}(y)=\lambda$. Thus

$$
\ln x_{i}=\sum_{j=1}^{n} a_{i j}\left(\ln y_{j}\right)=\sum_{j=1}^{n} \ln \left(y_{j}^{a_{i j}}\right)=\ln \left(\prod_{j=1}^{n} y_{j}^{a_{i j}}\right)
$$

i.e.

$$
x_{i}=\prod_{j=1}^{n} y_{j}^{a_{i j}} \text { and } x^{\alpha}=\prod_{j=1}^{n} y_{j}^{\left\langle a_{j}, \alpha\right\rangle},
$$

where $a_{j}=\left(a_{i j}\right)_{1 \leq i \leq n}$. From these considerations, we define

$$
\begin{aligned}
w:[0,1]^{n} & \rightarrow \quad[0,1]^{n} \\
y & \mapsto x=w(y), \quad \text { where } x_{i}=\prod_{j=1}^{n} y_{j}^{a_{i j}} .
\end{aligned}
$$


By using $w$ as a change of variables in $I_{\bar{\Delta}_{\tau}}(s)$, one gets

$$
I_{\bar{\Delta}_{\tau}}(s)=\left|\operatorname{det}\left[a_{1}, \ldots, a_{n}\right]\right|_{\mathbb{R}} \int_{[0,1]^{n}} \phi(y)\left(\prod_{j=1}^{n} y_{j}^{\sigma\left(a_{j}\right)-1}\right)|\boldsymbol{f}(w(y))|_{\mathbb{R}}^{s}|d y|,
$$

where $\operatorname{det}\left[a_{1}, \ldots, a_{n}\right]$ denotes the determinant of the matrix with columns $a_{1}, \ldots, a_{n}$ and $\phi(y):=\Phi(w(y))$.

6.1.2. Description of $w^{-1}\{0\}$. The set $w^{-1}\{0\}$ plays an important role in the considerations below. We give here some properties of this set, proven in [5, Lemma 5.1] and [5, Lemma 5.2], that we will use later on.

Lemma 4. (1) Take $y \in[0,1]^{n}$. Set $I=\left\{i \in\{1, \ldots, n\} \mid y_{i}=0\right\}$. Assume that $I \neq \emptyset$ and that $\tau^{\prime}=\cap_{i \in I} F\left(a_{i}\right) \neq \emptyset$. Then

$$
y \in w^{-1}\{0\} \text { if and only if } \tau^{\prime} \text { is a compact face of } \Gamma(\boldsymbol{f}) .
$$

(2) If $V$ is a small enough neighborhood (resp. a neighborhood) of the origin in $[0,1]^{n}$, then $w^{-1}(V)$ is a small enough neighborhood (resp. neighborhood) of $w^{-1}\{0\}$ in $[0,1]^{n}$.

6.1.3. Properties of $\boldsymbol{f} \circ w$. Writing $f_{i}=\sum_{m} c_{m, i} x^{m}$ we note for any $i=1, \ldots, l$ that

$$
f_{i}(w(y))=\sum_{m} c_{m, i}(w(y))^{m}=\sum_{m} c_{m, i} \prod_{j=1}^{n} y_{j}^{\left\langle a_{j}, m\right\rangle},
$$

what we can write as

$$
f_{i}(w(y))=\left(\prod_{j=1}^{n} y_{j}^{d\left(a_{j}\right)}\right) f_{i}^{*}(y)
$$

where $d\left(a_{j}\right)=\min _{x \in \Gamma(\boldsymbol{f})}\left\langle a_{j}, x\right\rangle$. If $\operatorname{supp}\left(f_{i}\right) \cap \tau \neq \emptyset$, then the minimum of all $\left\langle a_{j}, m\right\rangle$ is attained at $\tau$ and $f_{i}^{*}(0) \neq 0$. If $\operatorname{supp}\left(f_{i}\right) \cap \tau=\emptyset$, then $f_{i}^{*}(0)=0$.

From now on we pick a sufficiently small neighborhood $W$ of $w^{-1}\{0\}$ on which the series in the right hand side of (6.2) converges for every $i$. Then for every $i$ the restriction of this series to $W \cap[0,1]^{n}$ agrees with $f_{i} \circ w$.

6.2. Meromorphic Continuation of $I_{\bar{\Delta}_{\tau}}(s)$.

Theorem 5. Let $\boldsymbol{f}: U\left(\subset \mathbb{R}^{n}\right) \longrightarrow \mathbb{R}^{l}$, with $\boldsymbol{f}(0)=0$, be an analytic mapping, strongly non-degenerate at the origin with respect to $\Gamma(\boldsymbol{f})$. Assume that $\tau=\cap_{i=1}^{n} F\left(a_{i}\right)$ is a 0-dimensional face of $\Gamma(\boldsymbol{f})$, and that $\boldsymbol{f}$ is compatible with $\mathcal{L}^{-1}\left(\bar{\Delta}_{\tau}\right)$. There exists a neighborhood $V(\subset U)$ of the origin such that, if $\Phi$ is a smooth function with support contained in $V$, the following assertions hold.

(1) $I_{\bar{\Delta}_{\tau}}(s)$ is convergent and defines a holomorphic function of $s$ on $\operatorname{Re}(s)>$ $\max \left\{-l,-\frac{\sigma\left(a_{1}\right)}{d\left(a_{1}\right)}, \ldots,-\frac{\sigma\left(a_{n}\right)}{d\left(a_{n}\right)}\right\}$,

(2) $I_{\bar{\Delta}_{\tau}}(s)$ admits a meromorphic extension to the complex plane with poles of order at most $n$. Furthermore, the poles belong to

$$
\left(\bigcup_{1 \leq i \leq n}\left(-\frac{\sigma\left(a_{i}\right)+\mathbb{N}}{d\left(a_{i}\right)}\right)\right) \cup(-(l+\mathbb{N}))
$$


(3) Let $\rho$ be a positive integer and let $s_{0}$ be a candidate pole of $I_{\bar{\Delta}_{\tau}}(s)$, with $s_{0} \notin$ $-(l+\mathbb{N})$ (resp. $\left.s_{0} \in-(l+\mathbb{N})\right)$. A necessary condition for $s_{0}$ to be a pole of $I_{\bar{\Delta}_{\tau}}(s)$ of order $\rho$, is that

$$
\operatorname{Card}\left\{i \mid s_{0} \in\left(-\frac{\sigma\left(a_{i}\right)+\mathbb{N}}{d\left(a_{i}\right)}\right)\right\} \geq \rho(\text { resp. } \geq \rho-1) .
$$

Proof. We first prove (1) and (2). By (6.1) it is sufficient to prove the result for

$$
I_{\Omega}(s)=\int_{[0,1]^{n}} \Omega(y)\left(\prod_{i=1}^{n} y_{j}^{\sigma\left(a_{j}\right)-1}\right)|\boldsymbol{f}(w(y))|_{\mathbb{R}}^{s}|d y|,
$$

where $\Omega$ is a smooth function. By using a sufficiently fine partition of the unity, one can express $I_{\Omega}(s)$ as a finite sum of analogous integrals $I_{\Omega_{p}}(s)$, where $\Omega_{p}$ is a smooth function with support contained in a small ball around a point $p$ belonging to the support of $\Omega$. The relevant points $p$ to consider belong to $w^{-1}\{0\}$. In the sequel we may and will assume that $\operatorname{supp}\left(\Omega_{p}\right)$ is as small as necessary for the arguments that follow. Several cases occur.

Case 1 ( $p$ is the origin of $\mathbb{R}^{n}$ ).

Assume that $\Omega_{0}$ is a smooth function containing the origin. There exists at least one index $i_{0}$ such that $\operatorname{supp}\left(f_{i_{0}}\right) \cap \tau \neq \emptyset$. Hence, with the notation of (6.3), there exists a small neighborhood of the origin $V_{0, \tau}$ such that $\{0\} \subset \operatorname{supp}\left(\Omega_{0}\right) \subset V_{0, \tau}$, and $f_{i_{0}}^{*}(y) \neq 0$ for any $y \in V_{0, \tau}$. Consequently, we have that $\sum_{i=1}^{l}\left(f_{i}^{*}(y)\right)^{2}>0$ on $V_{0, \tau}$, and then by Lemma 1 the integral

$$
I_{\Omega_{0}}(s)=\int_{[0,1]^{n}} \Omega_{0}(y)\left(\prod_{j=1}^{n} y_{j}^{s d\left(a_{j}\right)+\sigma\left(a_{j}\right)-1}\right)\left(\sum_{i=1}^{l}\left(f_{i}^{*}(y)\right)^{2}\right)^{s / 2}|d y|
$$

has a meromorphic continuation to $\mathbb{C}$, with poles (of order at most $n$ ) belonging to

$$
\bigcup_{1 \leq i \leq n}\left(-\frac{\sigma\left(a_{i}\right)+\mathbb{N}}{d\left(a_{i}\right)}\right) \text {. }
$$

Case 2 ( $p$ has exactly $r$ coordinates equal to zero, with $1 \leq r \leq n-1$ ).

After renaming the variables, we may suppose that the first $r$ coordinates are zero, i.e. $p$ is the form $p=\left(0, \ldots, 0, p_{r+1}, \ldots, p_{n}\right)$. Let $\tau^{\prime}$ be the first meet locus of the cone $\Delta_{\tau^{\prime}}$, spanned by $a_{1}, \ldots, a_{r}$; it is a compact face of $\Gamma(\boldsymbol{f})$. Note that, similarly as in [1, Chap. II, $\S 8$, Lemme 9],

$$
f_{i}(w(y))=\prod_{j=1}^{r} y_{j}^{d\left(a_{j}\right)}\left(\widetilde{f}_{i}\left(y_{r+1}, \ldots, y_{n}\right)+O_{i}\left(y_{1}, \ldots, y_{n}\right)\right),
$$

where the $\tilde{f}_{i}$ are polynomials in $y_{r+1}, \ldots, y_{n}$ and the $O_{i}\left(y_{1}, \ldots, y_{n}\right)$ are analytic functions in $y_{1}, \ldots, y_{n}$ but belonging to the ideal generated by $y_{1}, \ldots, y_{r}$. Here $\tilde{f}_{i}$ is identically zero if and only if $\operatorname{supp}\left(f_{i}\right) \cap \tau^{\prime}=\emptyset$. Furthermore,

$$
f_{i, \tau^{\prime}}(w(y))=\prod_{j=1}^{r} y_{j}^{d\left(a_{j}\right)}\left(\widetilde{f}_{i}\left(y_{r+1}, \ldots, y_{n}\right)\right) .
$$

For the sequel we redefine $f_{i}^{*}(y)$ as

$$
f_{i}^{*}(y)=\tilde{f}_{i}\left(y_{r+1}, \ldots, y_{n}\right)+O_{i}\left(y_{1}, \ldots, y_{n}\right) .
$$


Set $\widetilde{p}=\left(p_{r+1}, \ldots, p_{n}\right) \in\left(\mathbb{R}^{\times}\right)^{n-r}$. To accomplish the proof of Case 2 , we need to study the following three subcases.

Subcase 2.1 (There exists an index $i$ such that $\tilde{f}_{i}(\widetilde{p}) \neq 0$ ). Then there exists a neighborhood $V_{p, \tau^{\prime}}$ of $p=\left(0, \ldots, 0, p_{r+1}, \ldots, p_{n}\right)$, such that $f_{i}^{*}(y) \neq 0$ for any $y \in V_{p, \tau^{\prime}}$ and such that $\operatorname{supp}\left(\Omega_{p}\right) \subset V_{p, \tau^{\prime}}$. Hence

$$
g\left(y_{1}, \ldots, y_{n}\right):=\left(\sum_{i=1}^{l}\left(f_{i}^{*}\left(y_{1}, \ldots, y_{n}\right)\right)^{2}\right)^{1 / 2}>0,
$$

for any $y \in V_{p, \tau^{\prime}}$, and, by Lemma 1 ,

$$
I_{\Omega_{p}}(s)=\int_{[0,1]^{n}} \Omega_{p}(y)\left(\prod_{j=1}^{r} y_{j}^{s d\left(a_{j}\right)+\sigma\left(a_{j}\right)-1}\right) g(y)^{s}|d y|
$$

has a meromorphic continuation to the whole complex plain with poles contained in

$$
\bigcup_{1 \leq i \leq r}\left(-\frac{\sigma\left(a_{j}\right)+\mathbb{N}}{d\left(a_{j}\right)}\right)
$$

Subcase $2.2\left(\tilde{f}_{i}(\widetilde{p})=0\right.$ for $i=1, \ldots, l$ and $\left.\widetilde{p} \in(0,1)^{n-r}\right)$. By the non-degeneracy condition, the fact that $w$ is an analytic isomorphism on $\left(\mathbb{R}_{+} \backslash\{0\}\right)^{n}$ and (6.7), one gets for any $y=\left(\left(y_{1}, \ldots, y_{r}\right), \widetilde{y}\right) \in\left(\mathbb{R}_{+} \backslash\{0\}\right)^{r} \times\left(\mathbb{R}_{+} \backslash\{0\}\right)^{n-r} \cap\left\{y \mid \tilde{f}_{1}(\tilde{y})=\cdots=\right.$ $\left.\widetilde{f}_{l}(\tilde{y})=0\right\}$ that

$$
\begin{gathered}
\operatorname{rank}\left[\frac{\partial f_{i, \tau^{\prime}}}{\partial y_{j}}(w(y))\right]= \\
\operatorname{rank}\left[\begin{array}{cccccc}
0 & \ldots & 0 & \prod_{j=1}^{r} y_{j}^{d\left(a_{j}\right)} \frac{\partial \widetilde{f}_{1}}{\partial y_{r+1}}(\tilde{y}) & \ldots & \prod_{j=1}^{r} y_{j}^{d\left(a_{j}\right)} \frac{\partial \widetilde{f}_{1}}{\partial y_{n}}(\tilde{y}) \\
\vdots & & \vdots & \vdots & & \vdots \\
0 & \ldots & 0 & \prod_{j=1}^{r} y_{j}^{d\left(a_{j}\right)} \frac{\partial \widetilde{f}_{l}}{\partial y_{r+1}}(\tilde{y}) & \ldots & \prod_{j=1}^{r} y_{j}^{d\left(a_{j}\right)} \frac{\partial \widetilde{f}_{l}}{\partial y_{n}}(\tilde{y})
\end{array}\right]=l .
\end{gathered}
$$

Now this implies for $\tilde{y}=\left(y_{r+1}, \ldots, y_{n}\right) \in\left(\mathbb{R}_{+} \backslash\{0\}\right)^{n-r} \cap\left\{y \mid \tilde{f}_{1}(\tilde{y})=\cdots=\widetilde{f}_{l}(\tilde{y})=\right.$ $0\}$ that

$$
\operatorname{rank}\left[\begin{array}{ccc}
\frac{\partial \widetilde{f}_{1}}{\partial y_{r+1}} & \cdots & \frac{\partial \widetilde{f}_{1}}{\partial y_{n}} \\
\vdots & \cdots & \vdots \\
\frac{\partial \widetilde{f}_{l}}{\partial y_{r+1}} & \cdots & \frac{\partial \widetilde{f}_{l}}{\partial y_{n}}
\end{array}\right](\widetilde{y})=l .
$$

Then necessarily $l \leq n-r$, all $\widetilde{f}_{i}$ are non-zero polynomials, and $\operatorname{rank}\left[\frac{\partial \widetilde{f}_{i}}{\partial y_{j}}(\widetilde{p})\right]=l$.

Since $\left[\frac{\partial \widetilde{f}_{i}}{\partial y_{j}}(\widetilde{p})\right]=\left[\frac{\partial\left(\widetilde{f}_{i}+O_{i}\right)}{\partial y_{j}}(p)\right]$, cf. (6.6), this last matrix having rank $l$ implies that we can choose new coordinates $y^{\prime}=\left(y_{1}, \ldots, y_{r}, y_{r+1}^{\prime}, \ldots, y_{r+l}^{\prime}, y_{r+l+1}, \ldots, y_{n}\right)$ in a neighborhood $V_{p}$ of $p$, with $\operatorname{supp}\left(\Omega_{p}\right) \subset V_{p}$, such that

$$
f_{i}\left(\omega\left(y^{\prime}\right)\right)=\prod_{j=1}^{r}\left(y_{j}^{\prime}\right)^{d\left(a_{j}\right)} y_{r+i}^{\prime}
$$


for $i=1, \ldots, l$ and hence

$$
|\boldsymbol{f}(w(y))|_{\mathbb{R}}^{s}=\left(\sum_{i=1}^{l} f_{i}^{2}\left(w\left(y^{\prime}\right)\right)\right)^{s / 2}=\prod_{j=1}^{r}\left(y_{j}^{\prime}\right)^{s d\left(a_{j}\right)}\left(\sum_{i=1}^{l}\left(y_{r+i}^{\prime}\right)^{2}\right)^{s / 2}
$$

Consequently

$$
I_{\Omega_{p}}(s)=\int \widetilde{\Omega}_{p}\left(y^{\prime}\right) \prod_{j=1}^{r}\left(y_{j}^{\prime}\right)^{s d\left(a_{j}\right)+\sigma\left(a_{j}\right)-1}\left(\sum_{i=1}^{l}\left(y_{r+i}^{\prime}\right)^{2}\right)^{s / 2}\left|d y^{\prime}\right|,
$$

where the integration is performed over $[0,1]^{r} \times U \times[0,1]^{n-l-r}$, where $U$ is a small neighborhood of the origin in $\mathbb{R}^{l}$. By applying Corollary 2, we get a meromorphic continuation to the whole complex plane for (6.10), with poles belonging to

$$
\left(\bigcup_{1 \leq i \leq r}\left(-\frac{\sigma\left(a_{j}\right)+\mathbb{N}}{d\left(a_{j}\right)}\right)\right) \cup(-(l+\mathbb{N}))
$$

Subcase $2.3\left(\tilde{f}_{i}(\widetilde{p})=0\right.$ for $i=1, \ldots, l$ and $\widetilde{p} \in(0,1]^{n-r}$ with at least one coordinate equal to 1$)$. By renaming the variables we may assume that $\widetilde{p}$ has the form $\widetilde{p}=\left(p_{r+1}, \ldots, p_{r+t}, 1, \ldots, 1\right)$, with $p_{r+i} \in(0,1)$ for each $i=1, \ldots, t$.

Claim. (1) If $0 \leq t \leq l-1$, there exists an index $i_{0}$ such that $f_{i_{0}}^{*}(p) \neq 0$.

(2) If $t \geq l$, then

$$
\operatorname{rank}\left[p_{j}\left(1-p_{j}\right) \frac{\partial f_{i}^{*}}{\partial y_{j}}(p)\right]_{\substack{1 \leq i \leq l \\ 1 \leq j \leq n}}=l
$$

Assuming the claim, we conclude when $0 \leq t \leq l-1$, by a similar argument as the one given for Case 1 , that $I_{\Omega_{p}}(s)$ has a meromorphic continuation to $\mathbb{C}$ with poles contained in the set (6.9). When $t \geq l$, we can choose new coordinates $y^{\prime}=\left(y_{1}, \ldots, y_{r}, y_{r+1}^{\prime}, \ldots, y_{r+l}^{\prime}, y_{r+l+1}, \ldots, y_{n}\right)$ in a neighborhood $V_{p}$ of $p$ such that $y_{r+i}^{\prime}=f_{i}^{*}(y)$ for $i=1, \ldots, l$. In this coordinate system $I_{\Omega_{p}}(s)$ has the form (6.10), and thus meromorphic continuation is obtained as in Subcase 2.2.

Proof of the claim. Set $I=\left\{i \mid p_{i}=0\right\}=\{1, \ldots, r\}, J=\left\{i \mid p_{i}=1\right\}=$ $\{r+t+1, \ldots, n\}$ and $K=\left\{i \mid 0<p_{i}<1\right\}=\{r+1, \ldots, r+t\}$. Set also

$$
A=\left\{y \in[0,1]^{n} \mid y_{j}=0 \text { if } j \in I, y_{j}=1 \text { if } j \in J, \text { and } 0<y_{j}<1 \text { if } j \in K\right\},
$$
and

$$
\widehat{A}=\left\{z \in[0,1]^{n} \mid z_{j}=1 \text { if } j \in I \cup J, \text { and } 0<z_{j}<1 \text { if } j \in K\right\} .
$$

Given $y \in A$, we denote by $\hat{y}$ the element of $\widehat{A}$ such that $y_{k}=\hat{y}_{k}$ for $k \in K$. Set $\Delta_{K}=\left\{\sum_{j \in K} \lambda_{j} a_{j} \mid \lambda_{j} \in \mathbb{R}_{+} \backslash\{0\}\right\}$ and $S_{K}=\mathcal{L}^{-1}\left(\Delta_{K}\right)$ as before.

If $0 \leq t=\operatorname{Card}(K) \leq l-1$, there exists an index $i_{0}$, such that $\widetilde{f}_{i_{0}}(p) \neq 0$, by the compatibility condition and (6.7). If $t=\operatorname{Card}(K) \geq l$, the compatibility condition asserts, for

$$
\begin{gathered}
\widehat{y}=\left(1, \ldots, 1, y_{r+1}, \ldots, y_{r+t}, 1, \ldots, 1\right) \\
\in\left\{\widehat{y} \in \widehat{A} \mid\left(f_{1, \tau^{\prime}} \circ w\right)(\widehat{y})=\cdots=\left(f_{l, \tau^{\prime}} \circ w\right)(\widehat{y})=0\right\}
\end{gathered}
$$

that

$$
\operatorname{rank}\left[\frac{\partial\left(f_{i, \tau^{\prime}} \circ w\right)}{\partial y_{j}}(\widehat{y})\right]_{\substack{1 \leq i \leq l \\ 1 \leq j \leq n}}=\operatorname{rank}\left[\frac{\partial\left(f_{i, \tau^{\prime}} \circ w\right)}{\partial y_{j}}(\widehat{y})\right]_{\substack{1 \leq i \leq l \\ j \in K}}=l .
$$


Note for the first equality that the restriction of $\omega$ to $\widehat{A}$ is a parametrization of $S_{K}$. By (6.6) and (6.7) this condition on $y$ is equivalent, for

$$
y=\left(0, \ldots, 0, y_{r+1}, \ldots, y_{r+t}, 1, \ldots, 1\right) \in\left\{y \in A \mid f_{1}^{*}(y)=\cdots=f_{l}^{*}(y)=0\right\},
$$

to

$$
\operatorname{rank}\left[\frac{\partial f_{i}^{*}}{\partial y_{j}}(y)\right]_{\substack{1 \leq i \leq l \\ j \in K}}=l
$$

what can be rewritten as

$$
\begin{gathered}
l=\operatorname{rank}\left[y_{j}\left(1-y_{j}\right) \frac{\partial f_{i}^{*}}{\partial y_{j}}(y)\right]_{\substack{1 \leq i \leq l \\
1 \leq j \leq n}}= \\
\operatorname{rank}\left[\begin{array}{ccccccccc}
0 & \ldots & 0 & y_{r+1}\left(1-y_{r+1}\right) \frac{\partial f_{1}^{*}}{\partial y_{r+1}}(y) & \ldots & y_{r+t}\left(1-y_{r+t}\right) \frac{\partial f_{1}^{*}}{\partial y_{r+t}}(y) & 0 & \ldots & 0 \\
\vdots & & \vdots & \vdots & \vdots & \vdots & & \vdots \\
0 & \ldots & 0 & y_{r+1}\left(1-y_{r+1}\right) \frac{\partial f_{l}^{*}}{\partial y_{r+1}}(y) & \ldots & y_{r+t}\left(1-y_{r+t}\right) \frac{\partial f_{l}^{*}}{\partial y_{r+t}}(y) & 0 & \ldots & 0
\end{array}\right] .
\end{gathered}
$$

This finishes the proof of the claim.

The third part of the theorem follow from (6.5), (6.8) and (6.10) by applying Lemma 1 and Corollary 2.

Finally we state in the following theorem the meromorphic continuation of

$$
I(s)=I(s, \boldsymbol{f}, \Phi)=\int_{\mathbb{R}_{+}^{n}} \Phi(x)|\boldsymbol{f}(x)|_{\mathbb{R}}^{s}|d x| .
$$

Recall the notation

$$
\mathcal{P}(\xi)=\left\{-\frac{\sigma(\xi)+k}{d(\xi)} \mid k \in \mathbb{N}\right\}
$$

for $\xi \in \mathfrak{D}(\Gamma(f))$.

Theorem 6. Let $\boldsymbol{f}: U\left(\subset \mathbb{R}^{n}\right) \longrightarrow \mathbb{R}^{l}$, with $\boldsymbol{f}(0)=0$, be an analytic mapping, strongly non-degenerate at the origin with respect to $\Gamma(\boldsymbol{f})$. There exists a neighborhood $V(\subset U)$ of the origin such that, if $\Phi$ is a smooth function with support contained in $V$, the following assertions hold:

(1) $I(s)$ converges and defines a holomorphic function on $\operatorname{Re}(s)>\max \left\{-\gamma_{0}(\boldsymbol{f}),-l\right\}$, with

$$
\gamma_{0}(\boldsymbol{f})=\min _{\xi \in \mathfrak{D}(\Gamma(\boldsymbol{f}))}\left\{\frac{\sigma(\xi)}{d(\xi)}\right\},
$$

(2) I(s) admits a meromorphic continuation to the whole complex plane with poles of order at most $n$, and the poles belong to

$$
\left(\bigcup_{\xi \in \mathfrak{D}(\Gamma(\boldsymbol{f}))} \mathcal{P}(\xi)\right) \cup(-(l+\mathbb{N})) .
$$

(3) Let $\rho$ be a positive integer and let $s_{0}$ be a candidate pole of $I(s)$, with $s_{0} \notin$ $-(l+\mathbb{N})\left(\right.$ resp. $\left.s_{0} \in-(l+\mathbb{N})\right)$. A necessary condition for $s_{0}$ to be a pole of $I(s)$ or order $\rho$, is that there exists a face $\tau$ of $\Gamma(\boldsymbol{f})$, of codimension $\rho$ (resp. $\rho-1)$, such that $s_{0} \in \mathcal{P}(\xi)$ for all facets of $\Gamma(\boldsymbol{f})$ containing $\tau$. 
Proof. Pick a simplicial fan $\mathcal{F}_{\boldsymbol{f}}$ subordinated to $\Gamma(\boldsymbol{f})$. By Lemma 3 there exists $b \in\left(\mathbb{R}_{+} \backslash\{0\}\right)^{n}$ such that $\boldsymbol{f} \circ T_{b}$ is compatible with $\mathcal{F}_{\boldsymbol{f}}$. Since the change of variables $x=T_{b}(y)$ does not affect the meromorphic continuation of $I(s)$, we may assume that $\boldsymbol{f}$ is compatible with the decoupage $\mathcal{L}^{-1}\left(\mathcal{F}_{\boldsymbol{f}}\right)$. Parts (1) and (2) follow immediately from Theorem 5 by the decomposition

$$
I(s)=\int_{\mathbb{R}_{+}^{n}} \Phi(x)|\boldsymbol{f}(x)|_{\mathbb{R}}^{s} d x=\sum_{\bar{\Delta}_{\tau} \in \mathcal{F}} I_{\bar{\Delta}_{\tau}}(s) .
$$

Part (3) also follows from Theorem 5 by exactly the same argument as in the proof of [5, Théorème 6.1 (iii)], using [5, Corollaire 2.2].

\section{REFERENCES}

[1] Arnold V. I., Gussein-Zade S. M., Varchenko A. N., Singularités des applications différentiables, Vol II, Éditions Mir, Moscou, 1986.

[2] Atiyah M. F., Resolution of singularities and division of distributions. Comm. Pure Appl. Math. 231970 145-150.

[3] Bernstein I. N. and Gel'fand S. I., Meromorphic property of the functions $P^{\lambda}$, Functional. Anal. Appl., 3 (1969), 68-69.

[4] Bernstein I. N., The analytic continuation of generalized functions with respect to a parameter, Functional. Anal. Appl., 6 (1972), 273-285.

[5] Denef J. and Sargos P., Polyèdre de Newton et distribution $f_{+}^{s}$. I, J. Analyse Math., 53 (1989), 201-218.

[6] Ein L. and Mustaţă M., Invariants of singularities of pairs, Proc. International Congress of Mathematicians 2006, Vol. II, 583-602, Eur. Math. Soc., Zürich, 2006.

[7] Encinas S., Nobile A. and Villamayor O., On algorithmic equi-resolution and stratification of Hilbert schemes, Proc. London Math. Soc. 86 (2003), no. 3, 607-648.

[8] Gel'fand I. M. and Shilov G.E., Generalized Functions, vol 1., Academic Press, New York and London, 1977.

[9] Hironaka H., Resolution of singularities of an algebraic variety over a field of characteristic zero, Ann. Math. 79 (1964), 109-326.

[10] Igusa J.-I., Complex powers and asymptotic expansions, I. Functions of certain types, J. Reine Angew. Math. 268/269 (1974), 110-130; II. Asymptotic expansions, J. Reine Angew. Math. 278/279 (1975), 307-321.

[11] Igusa J.-I., Forms of higher degree. Tata Institute of Fundamental Research Lectures on Mathematics and Physics, 59. Tata Institute of Fundamental Research, Bombay; by the Narosa Publishing House, New Delhi, 1978. iv+175 pp.

[12] Igusa J.-I., Some aspects of the arithmetic theory of polynomials. Discrete groups in geometry and analysis (New Haven, Conn., 1984), 20-47, Progr. Math., 67, Birkhäuser Boston, Boston, MA, 1987.

[13] Igusa J.-I., An introduction to the theory of local zeta functions, AMS/IP Studies in Advanced Mathematics, 2000.

[14] Kempf G., Knudsen F., Mumford D. and Saint-Donat B., Toroidal embeddings, Lectures notes in Mathematics vol. 339, Springer-Verlag, 1973.

[15] Khovanskii A. G., Newton polyhedra and toroidal varieties, Funct. Anal. Appl. 12 (1978), no.1, 51-61.

[16] Kollár J., Singularities of pairs, Summer Research Institute on Algebraic Geometry (Santa Cruz 1995), Amer. Math. Soc., Proc. Symp. Pure Math. 62, Part 1 (1997), 221-287.

[17] Langlands R. P., Orbital integrals on forms of $S L(3)$. I. Amer. J. Math. 105 (1983), no. 2, 465-506.

[18] Phong D. H., Sturm J., Algebraic estimates, stability of local zeta functions, and uniform estimates for distribution functions, Ann. of Math. (2) 152 (2000), no. 1, 277-329.

[19] Saito M., On real log canonical thresholds, arXiv:0707.2308v3 [math.AG].

[20] Varchenko A., Newton polyhedra and estimation of oscillating integrals, Funct. Anal. Appl. 10 (1976), 175-196. 
[21] Veys W. and Zúñiga-Galindo W. A., Zeta functions associated with polynomial mappings, log-principalization, Trans. Amer. Math. Soc. 360 (2008), 2205-2227.

[22] Włodarczyk J., Simple Hironaka resolution in characteristic zero, J. Amer. Math. Soc. 18 (2005), 779-822.

Centro de Investigación y de Estudios Avanzados del Instituto Politécnico Nacional, Departamento de Matemáticas- Unidad Querétaro, Libramiento Norponiente \#2000, Fracc. Real de Juriquilla. Santiago de Querétaro, Qro. 76230, México.

E-mail address: eleon@math.cinvestav.mx

University of Leuven, Department of Mathematics, Celestijnenlaan 200 B, B-3001 Leuven (Heverlee), Belgium

E-mail address: wim.veys@wis.kuleuven.be

Centro de Investigación y de Estudios Avanzados del Instituto Politécnico Nacional, Departamento de Matemáticas- Unidad Querétaro, Libramiento Norponiente \#2000, Fracc. Real de Juriquilla. Santiago de Querétaro, Qro. 76230, México.

E-mail address: wazuniga@math.cinvestav.edu.mx 